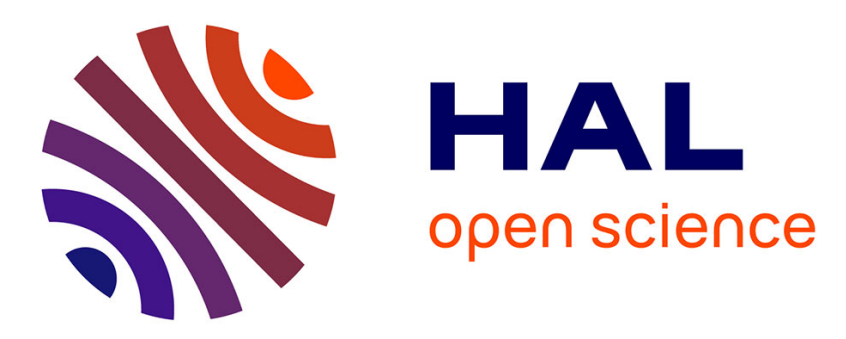

\title{
The Binary Companion of Young, Relativistic Pulsar $\mathrm{J} 1906+0746$
}

Joeri van Leeuwen, Laura Kasian, Ingrid H. Stairs, D. R. Lorimer, F. Camilo, S. Chatterjee, Ismaël Cognard, G. Desvignes, P. C. C. Freire, G. H. Janssen, et al.

\section{To cite this version:}

Joeri van Leeuwen, Laura Kasian, Ingrid H. Stairs, D. R. Lorimer, F. Camilo, et al.. The Binary Companion of Young, Relativistic Pulsar J1906+0746. The Astrophysical journal letters, 2015, 798 (118), pp.15. 10.1088/0004-637X/798/2/118 . insu-01256048

\section{HAL Id: insu-01256048 https://hal-insu.archives-ouvertes.fr/insu-01256048}

Submitted on 9 Jun 2016

HAL is a multi-disciplinary open access archive for the deposit and dissemination of scientific research documents, whether they are published or not. The documents may come from teaching and research institutions in France or abroad, or from public or private research centers.
L'archive ouverte pluridisciplinaire HAL, est destinée au dépôt et à la diffusion de documents scientifiques de niveau recherche, publiés ou non, émanant des établissements d'enseignement et de recherche français ou étrangers, des laboratoires publics ou privés. 


\title{
THE BINARY COMPANION OF YOUNG, RELATIVISTIC PULSAR J1906+0746
}

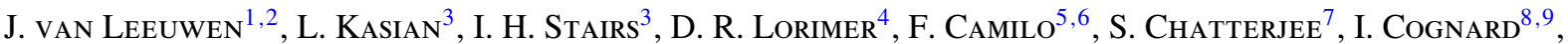

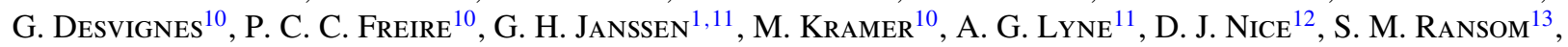 \\ B. W. STAPPERS ${ }^{11}$, AND J. M. WeisBerG ${ }^{14}$ \\ ${ }^{1}$ ASTRON, The Netherlands Institute for Radio Astronomy, Postbus 2, 7990 AA Dwingeloo, The Netherlands; leeuwen@ astron.nl \\ ${ }^{2}$ Astronomical Institute Anton Pannekoek, University of Amsterdam, Science Park 904, 1098 XH Amsterdam, The Netherlands \\ ${ }^{3}$ Department of Physics and Astronomy, University of British Columbia, Vancouver, BC V6T 1Z1, Canada \\ ${ }^{4}$ Department of Physics, West Virginia University, Morgantown, WV 26506, USA \\ ${ }_{5}^{5}$ Arecibo Observatory, HC3 Box 53995, Arecibo, PR 00612, USA \\ ${ }^{6}$ Columbia Astrophysics Laboratory, Columbia University, New York, NY 10027, USA \\ ${ }^{7}$ Center for Radiophysics and Space Research, Cornell University, Ithaca, NY 14853, USA \\ ${ }^{8}$ Laboratoire de Physique et Chimie de l'Environnement et de l'Espace LPC2E CNRS-Université d'Orléans, F-45071 Orléans, France \\ ${ }^{9}$ Station de radioastronomie de Nançay, Observatoire de Paris, CNRS/INSU F-18330 Nançay, France \\ ${ }^{10}$ Max-Planck-Institut für Radioastronomie, D-53121 Bonn, Germany \\ ${ }^{11}$ Jodrell Bank Centre for Astrophysics, School of Physics and Astronomy, University of Manchester, Manchester M13 9PL, UK \\ 12 Department of Physics, Lafayette College, Easton, PA 18042, USA \\ ${ }^{13}$ NRAO (National Radio Astronomy Observatory), Charlottesville, VA 22903, USA \\ ${ }^{14}$ Department of Physics and Astronomy, Carleton College, Northfield, MN 55057, USA \\ Received 2014 February 3; accepted 2014 November 4; published 2015 January 8
}

\begin{abstract}
PSR J1906+0746 is a young pulsar in the relativistic binary with the second-shortest known orbital period, of $3.98 \mathrm{hr}$. We here present a timing study based on five years of observations, conducted with the five largest radio telescopes in the world, aimed at determining the companion nature. Through the measurement of three post-Keplerian orbital parameters, we find the pulsar mass to be 1.291(11) $M_{\odot}$, and the companion mass $1.322(11) M_{\odot}$, respectively. These masses fit well in the observed collection of double neutron stars (DNSs), but are also compatible with other systems where a young pulsar such as J1906+0746 is orbited by a white dwarf (WD). Neither radio pulsations nor dispersion-inducing outflows that could have further established the companion nature were detected. We derive an $\mathrm{H}$ I-absorption distance, which indicates that an optical confirmation of a WD companion is very challenging. The pulsar is fading fast due to geodetic precession, limiting future timing improvements. We conclude that the young pulsar J1906+0746 is likely part of a DNS, or is otherwise orbited by an older WD, in an exotic system formed through two stages of mass transfer.
\end{abstract}

Key words: binaries: close - pulsars: individual (PSR J1906+0746) - stars: neutron - white dwarfs

\section{INTRODUCTION}

Binaries harboring a neutron star (NS) are windows on dynamical star systems that have undergone and survived at least one supernova. By precisely measuring pulsar times of arrival (TOAs), and fitting binary models to these, one can describe the orbital motions of the pulsars and their companions - and hence constrain their masses. In combination with other information such as the pulsar spin, orbit, and companion nature, these mass estimates can elucidate the binary interaction and mass transfer history.

In the vast majority of observed binary pulsar systems, the pulsar is the first-born compact object: there, it is found to have been spun up by accretion from its companion to a higher spin rate than seen in young pulsars. These spun-up pulsars have far lower magnetic fields than the general pulsar population. They thus show very stable rotation and evolve only very slowly-resulting in higher characteristic ages of $\sim 10$ Gyr. This stability and longevity means such recycled pulsars are observable for much longer periods of time than the high-field fast-evolving young pulsars.

The amount of recycling is related to the binary type: lowmass binary pulsar systems host millisecond pulsars (MSPs), which have spin periods of about $1-10 \mathrm{~ms}$, and are orbited by a low-mass white dwarfs (WDs). Pulsars with more massive white dwarf (WD) companions or NS companions, generally have longer spin periods (10-200 ms), and lower characteristic ages (Lorimer 2008). This is consistent with a spin-up picture in which the amount of mass transferred from the companion to the pulsar depends largely on the duration of the mass-transfer stage, and hence, on the mass of the progenitor of the companion (Alpar et al. 1982).

The $144 \mathrm{~ms}$ pulsar J1906+0746, discovered in precursor PALFA observations in 2004 (Cordes et al. 2006; van Leeuwen et al. 2006; Lorimer et al. 2006), is one of only a handful of known double-degenerate relativistic binaries where the pulsar is believed to be the younger of the two compact objects. Other such pulsars, listed in Table 1, include the WD binaries $\mathrm{J} 1141-6545$ and $\mathrm{B} 2303+46$, and the second pulsar in the double pulsar system, J0737-3039B. Pulsar J1906+0746 is in a $3.98 \mathrm{hr}$ binary orbit with an eccentricity of 0.085 , making it the relativistic binary pulsar with the second-shortest known orbital period-second only to the $2.4 \mathrm{hr}$ orbit double pulsar J0737-3039 (Table 1).

Data taken in 1998 and 2005 at Parkes showed rapid evolution (Lorimer et al. 2006, and Figure 1). Because there was no sign of mode changing behavior in the dense sampling after the discovery, and thus no sign of magnetospheric instabilities (e.g., Lyne et al. 2010), these profile changes indicated a change in the pulsar-observer viewing geometry. Such year timescale profile changes are likely due to geodetic precession, the general relativistic effect that causes spinning objects to 
Table 1

Known Pulsars in Relativistic and/or Double Neutron Star Binary Systems, Ordered by Pulsar Age, with Minor Ordering on Binary Period

\begin{tabular}{|c|c|c|c|c|c|c|}
\hline Pulsar & $\begin{array}{l}\text { Period } \\
(\mathrm{ms})\end{array}$ & $\begin{array}{c}P_{b} \\
\text { (days) }\end{array}$ & Eccentricity & $\begin{array}{c}\text { Pulsar } \\
\text { Mass }\left(M_{\odot}\right)\end{array}$ & $\begin{array}{l}\text { Companion } \\
\text { Mass }\left(M_{\odot}\right)\end{array}$ & $\begin{array}{c}\text { Companion } \\
\text { Type }\end{array}$ \\
\hline \multicolumn{7}{|c|}{ Young Pulsars in Relativistic Binaries } \\
\hline $\mathrm{J} 0737-3039 \mathrm{~B}^{1}$ & 2773.5 & 0.102 & 0.088 & $1.2489_{-0.0007}^{+0.0007}$ & $1.3381_{-0.0007}^{+0.0007}$ & NS \\
\hline $\mathrm{J} 1906+0746^{2}$ & 144.1 & 0.166 & 0.085 & $1.291_{-0.011}^{+0.011}$ & $1.322_{-0.011}^{+0.011}$ & NS or WD \\
\hline $\mathrm{J} 1141-6545^{3}$ & 393.9 & 0.198 & 0.17 & $1.27_{-0.01}^{+0.01}$ & $1.02_{0.01}^{+0.01}$ & WD \\
\hline B $2303+46^{4}$ & 1066.4 & 12.3 & 0.66 & $1.34_{-0.10}^{+0.10}$ & $1.3_{-0.10}^{+0.10}$ & WD \\
\hline \multicolumn{7}{|c|}{ Recycled Pulsars in Relativistic Double Neutron Star Binaries } \\
\hline J0737-3039A ${ }^{1}$ & 22.7 & 0.102 & 0.088 & $1.3381_{-0.0007}^{+0.0007}$ & $1.2489_{-0.0007}^{+0.0007}$ & NS \\
\hline $\mathrm{J} 1756-2251^{5}$ & 28.5 & 0.320 & 0.18 & $1.341_{-0.007}^{+0.007}$ & $1.230_{-0.007}^{+0.007}$ & NS \\
\hline $\mathrm{B} 1913+16^{6}$ & 59.0 & 0.323 & 0.62 & $1.439_{-0.0002}^{+0.0002}$ & $1.3886_{-0.0002}^{+0.0002}$ & NS \\
\hline $\mathrm{B} 2127+11 \mathrm{C}^{7}$ & 30.5 & 0.335 & 0.68 & $1.358_{-0.010}^{+0.010}$ & $1.354_{0.010}^{0.010}$ & NS \\
\hline B1534+12 & 37.9 & 0.421 & 0.27 & $1.3330_{-0.0002}^{+0.0002}$ & $1.3455_{-0.0002}^{+0.0002}$ & NS \\
\hline \multicolumn{7}{|c|}{ Recycled Pulsars in Long-Period ( $P_{b}>1$ day) Double Neutron Star Binaries } \\
\hline $\mathrm{J} 1518+4904^{9}$ & 40.9 & 8.63 & 0.25 & $0.72_{-0.58}^{+0.51}$ & $2.00_{-0.51}^{+0.58}$ & NS \\
\hline \multicolumn{7}{|c|}{ Total Mass $\left(M_{\odot}\right)$} \\
\hline $\mathrm{J} 1829+2456^{10}$ & 41.0 & 1.18 & 0.14 & \multicolumn{2}{|c|}{$2.5_{-0.2}^{+0.2}$} & NS \\
\hline $\mathrm{J} 1753-2240^{11}$ & 95.1 & 13.6 & 0.30 & \multicolumn{2}{|c|}{ Not measured } & NS \\
\hline $\mathrm{J} 1811-1736^{12}$ & 104.2 & 18.8 & 0.82 & \multicolumn{2}{|c|}{$2.57_{-0.10}^{+0.10}$} & NS \\
\hline
\end{tabular}

Note. Values from: ${ }^{1}$ Kramer et al. 2006; ${ }^{2}$ this work; ${ }^{3}$ Bhat et al. $2008 ;{ }^{4}$ Thorsett et al. 1993, Kulkarni \& van Kerkwijk $1998 ;{ }^{5}$ Ferdman et al. 2014; ${ }^{6}$ Weisberg et al. 2010; ${ }^{7}$ Jacoby et al. $2006 ;{ }^{8}$ Fonseca et al. 2014; ${ }^{9}$ Janssen et al. $2008 ;{ }^{10}$ Champion et al. $2005 ;{ }^{11}$ Keith et al. 2009; ${ }^{12}$ Corongiu et al. 2007.

precess about the total angular momentum vector of the system (Damour \& Ruffini 1974), an effect seen in other binary pulsars (J0737-3039B, Breton et al. 2008; J1141-6545, Hotan et al. 2005; B1534+12, Stairs et al. 2004; and B1913+16, Weisberg et al. 1989; Kramer 1998).

Given its high spin-down rate $\left(\dot{P} \sim 2 \times 10^{-14} \mathrm{~s} / \mathrm{s}\right)$ and short period, J1906+0746 is a young pulsar. Its characteristic age $\tau_{c}$ is roughly $112 \mathrm{kyr}$, the lowest of all known binary pulsars, and in the eighth percentile of the general pulsar age distribution (Manchester et al. 2005). Young pulsars show rapid spin-down evolution. By definition, this early $10^{5} \mathrm{yr}$ stage is much more fleeting than the $10^{9} \mathrm{yr}$ detectable life span of recycled pulsars. In binary systems, the recycled pulsars are thus common, the young pulsars rare. The recycled pulsars are generally the older of the two binary components-but the young pulsars formed more recently than their compact-object companion. Therefore, these latter provide a perspective on binary evolution that is different from the typical recycling scenario.

Pulsar J1906+0746 was intensively monitored with the Arecibo, Green Bank, Nançay, Jodrell Bank, and Westerbork radio telescopes, up to 2009 , after which the ever-decreasing pulse flux density (Figure 1) generally prevented significant further detections. We here present the high-precision followup timing from these telescopes over that initial five year period, 2005-2009.

Through this analysis, we are able to significantly improve the system characterization presented in the Lorimer et al. (2006) discovery paper: we measured two more post-Keplerian orbital parameters (for a total of three) - which, assuming general relativity, provide an over-constrained determination of the pulsar and companion masses.

In Section 2, we report on the observing and basic data reduction methods. Results on a measurement of the distance to J1906+0746 through its $\mathrm{HI}$ absorption are presented in Section 3. In Sections 4-7, we next detail our timing ap-

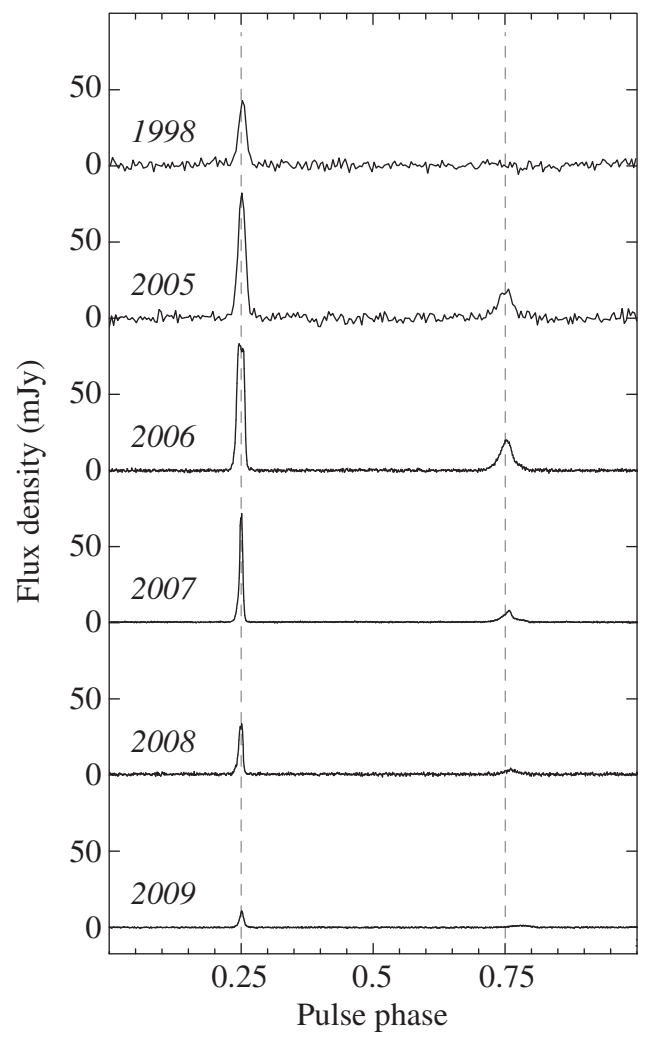

Figure 1. Profile evolution of J1906+0746. Shown are the 1998 and 2005 Parkes profiles (Lorimer et al. 2006), and yearly Arecibo profiles (this work). All subplots are the same scale. The 1998 and 2005 profiles used a different recording setup, with somewhat higher noise.

proach and methods, and the results from this timing campaign. We discuss the implications of these measurements in Sections 8 and 9 . 
Table 2

Details of the Telescope and Backend Setup

\begin{tabular}{|c|c|c|c|c|c|c|c|c|}
\hline Telescope & Backend & Epoch & Cadence Interval & $\begin{array}{c}\text { Central Frequency } \\
(\mathrm{MHz})\end{array}$ & $\begin{array}{c}\text { Bandwidth } \\
(\mathrm{MHz})\end{array}$ & $\begin{array}{c}\text { Channel BW } \\
(\mathrm{MHz})\end{array}$ & $\begin{array}{c}\text { Coherent } \\
\text { Dedispersion }\end{array}$ & $\mathbf{N}_{\text {TOAs }}$ \\
\hline \multirow[t]{2}{*}{ Arecibo } & WAPP & 2005-2009 & Week-month & $1170,1370,1570$ & $3 \times 100$ & 0.195 & $\mathrm{~N}$ & 23250 \\
\hline & $\mathrm{ASP}^{\mathrm{b}}$ & 2005-2009 & Week-month & $1420 / 1440$ & $16-32$ & 4 & $\mathrm{Y}$ & 220 \\
\hline \multirow[t]{2}{*}{ GBT } & $\mathrm{GASP}^{\mathrm{b}}$ & 2006-2009 & Week-month & 1404 & 64 & 4 & $\mathrm{Y}$ & 1110 \\
\hline & Spigot $^{\mathrm{c}}$ & $\ldots$ & $\ldots$ & 1440 & 600 & 0.781 & $\mathrm{~N}$ & $\ldots$ \\
\hline Nançay & $\mathrm{BON}^{\mathrm{d}}$ & 2005-2009 & Week & 1398 & 64 & 4 & $\mathrm{Y}$ & 650 \\
\hline \multirow[t]{2}{*}{ WSRT } & $\mathrm{PuMa}^{\mathrm{f}}$ & 2006-2007 & Month & 1380 & 80 & 0.156 & $\mathrm{~N}$ & 40 \\
\hline & $\mathrm{PuMaII}^{\mathrm{g}}$ & 2007-2009 & Month & 1380 & 160 & 0.313 & $\mathrm{Y}$ & 20 \\
\hline
\end{tabular}

Notes. $N_{\text {TOAs }}$ marks the number of TOAs generated per backend. Spigot data was used for searching, not for timing.

${ }^{\mathrm{a}}$ Dowd et al. (2000); ${ }^{\mathrm{b}}$ Demorest (2007) and Ferdman (2008); ${ }^{\mathrm{c}}$ Kaplan et al. (2005); ${ }^{\mathrm{d}}$ Desvignes (2009); ${ }^{\mathrm{e}}$ Hobbs et al. (2004); ${ }^{\mathrm{f}}$ Voûte et al. (2002); g Karuppusamy et al. (2008).

\section{OBSERVATIONS AND INITIAL DATA REDUCTION}

For the timing follow-up of this pulsar, we have obtained high signal-to-noise (S/N) data using the Arecibo Telescope and the Green Bank Telescope (GBT), covering several full orbits with the latter. High-cadence data from the Nançay, Jodrell Bank, and Westerbork telescopes provided further long-term timing coverage.

Data from the Arecibo telescope (USA) were taken using two backends simultaneously, as detailed in Table 2. Three Wideband Arecibo Pulsar Processor (WAPP) filterbank machines autocorrelated the two polarization channels. Offline, these were converted to spectra, dedispersed incoherently, summed, and finally folded at the local value of the pulsar period. Further data were taken using the Arecibo Signal Processor (ASP) coherent dedispersion machine, which folded on-line using the best-known values for the dispersion measure (DM) and the local pulse period. Through its coherent dedispersion capabilities, ASP is complementary to the WAPPs with their larger bandwidths. Parallel to this on-line folding, ASP recorded the $4 \mathrm{MHz}$ wide band around $1420 \mathrm{MHz}$ and stored the baseband data on disk, for an off-line investigation of $\mathrm{H}$ I absorption toward the pulsar (Section 3).

Data from the GBT (USA) were collected with the Pulsar Spigot card and, in parallel, the Green Bank Astronomical Signal Processor (GASP) coherent dedispersion machine, which is a GBT clone of ASP.

At the Nançay telescope (France), the Berkeley-OrléansNançay (BON) coherent dedispersion machine produced dedispersed and folded profiles every two minutes.

Data taken with the Lovell Telescope at the Jodrell Bank Observatory (UK) and its analog filterbank (AFB) were dedispersed and folded on-line at the nominal pulsar period.

At the Westerbork Synthesis Radio Telescope (WSRT) in The Netherlands, data was initially recorded with the pulsar machine PuMa. Starting in 2007, successor machine PuMaII added coherent dedispersion and doubled the bandwidth (Table 2).

\subsection{Flux Calibration and Offline Refolding}

The WAPP, ASP, and GASP data were flux-calibrated using the noise diode signal that was injected into the receiver, for each polarization individually, before each observation. When good calibration observations were not available, we normalized the flux density in each profile by the rms across the profile for the coherently dedispersed profiles, while weighting all channels equally for the WAPP filterbank data. A continuum source was used to further calibrate the ASP and GASP data for a significant portion of the epochs, using ASPFitsReader (Ferdman 2008). The Wideband Arecibo Pulsar Processor (WAPP) data were calibrated using SIGPROC ${ }^{15}$ and pre-recorded calibrator data. ${ }^{16}$

For all Arecibo and GBT data, time-averaged pulse profiles were finally created by adding both polarizations, all frequency channels, and five minute integrations. These profiles were remade whenever sufficient new Arecibo and GBT data were obtained to compute a new ephemeris. As the coherently dedispersed ASP and GASP data were recorded as a series of 30 or 60 second integrated pulse profiles, these were subsequently realigned to create new five minute integrated profiles.

The Jodrell Bank and Westerbork/PuMa profiles were produced using their respective custom off-line software, while the Nançay and the Westerbork/PuMaII profiles were reduced using the PSRCHIVE software package ${ }^{17}$ (Hotan et al. 2004), These data were not flux-calibrated; they were normalized by the rms of the noise.

\section{H I ABSORPTION IN THE PULSAR SPECTRUM}

Lorimer et al. (2006) combined the measured DM of $218 \mathrm{~cm}^{-3}$ pc with the NE2001 Galactic electron-density model (Cordes \& Lazio 2002) to estimate the distance to J1906+0746 of $5.40 \mathrm{kpc}$. Comparisons of NE2001 and Very Long Baseline Interferometry (VLBI) distances suggest an error of $20 \%$ (Deller et al. 2009b, although selection effects favoring nearby pulsars may lead to much larger errors in several cases), producing an overall estimate of $5.40_{-0.60}^{+0.56} \mathrm{kpc}$. Refining that estimate would be beneficial for several lines of follow-up analysis: it would improve our estimate of the kinematic contribution to the observed orbital period decay $\dot{P}_{b}$, and thus strengthen our general relativity tests (Section 5.3.2). It would also influence the likeliness of detecting a WD companion in the optical (Section 8).

Two more methods for distance determination exist, beyond the dispersion measure (DM) based method. These are based on parallax or kinematic measurements (for an in-depth comparison of these three methods, see Frail \& Weisberg 1990).

Precise parallactic distances through VLBI are now known for an increasing number of pulsars (e.g., Chatterjee et al. 2009), and at a nominal distance of $5.4 \mathrm{kpc}, \mathrm{J} 1906+0746$ is, in principle, nearby enough for such a measurement. However,

\footnotetext{
15 http://sigproc.sourceforge.net/

16 http://www.naic.edu/ phil/cals/cal.datR5

17 http://psrchive.sourceforge.net/
} 
the measurement is best performed with in-beam calibrators, and the process of identifying such sources was extremely laborious during the period in which $\mathrm{J} 1906+0746$ was, at $0.55 \mathrm{mJy}$ mean flux density, bright enough for observation with the Very Long Baseline Array (VLBA). While the process of identifying in-beam calibrators has been significantly streamlined at the VLBA with multi-phase center correlation (Deller et al. 2011), J1906+0746 had faded down to a mean flux density of $33 \mu \mathrm{Jy}$ in 2012, making it unsuitable for VLBA astrometry.

In the kinematic method, the absorption of pulsar emission by Doppler-shifted neutral hydrogen (H I) along the line of sight, can, once combined with a model of the Galactic kinematics, constrain the distance to the pulsar. In the off-pulse phases, the $\mathrm{H}_{\mathrm{I}}$ in its line of sight shines in emission; but during the onpulse phases, some of the pulsar's broadband emission will be absorbed by the Hi clouds between it and Earth (the "pulsar off" and "pulsar on" simulated spectra in Figure 2, left). As this absorption occurs at the Doppler-shifted H I frequency, it can be associated with a velocity relative to the observer. A Galactic rotation curve in the line of sight can then model at which distances from Earth such a velocity is expected. If the measured absorption velocities unambiguously map onto the curve, a lower limit to the pulsar distance can be produced. In some cases, an upper limit on the pulsar distance can also be derived: if certain features in the emission spectrum do not have corresponding absorption dips, the pulsar can be assumed to lie in front of the emitting region corresponding to the feature velocity (see Frail \& Weisberg 1990 and Weisberg et al. 2008 for further discussion).

Deriving a kinematic distance to J1906+0746 is especially interesting as its $D M$-derived distance is $5.40_{-0.60}^{+0.56} \mathrm{kpc}$, near the tangent point in this direction, at $6.4 \mathrm{kpc}$. The absence of absorption at the highest velocities, for example, would firmly put J1906+0746 closer to us than this tangent point.

Most kinematic $\mathrm{H}$ I distances have been determined for bright, slow pulsars (e.g., Frail \& Weisberg 1990; Johnston et al. 2001). Of the 70 pulsars with kinematic distances listed in Verbiest et al. (2012, their Table 1, excluding distances derived from associations), J1906+0746 is the eighth fastest spinning and, at $0.55 \mathrm{mJy}$, the dimmest. Combined with the high DM, measurement of $\mathrm{HI}$ absorption is challenging. We therefore used, for the first time in a pulsar H I absorption measurement, coherent dedispersion to maximize the $\mathrm{S} / \mathrm{N}$ by eliminating smearing between on- and off-pulse bins.

\subsection{Observations and Analysis}

During four full tracks on J1906+0746 with Arecibo, on 2006 June 14, July 11, October 12, and November 12, we recorded a total of $7.6 \mathrm{hr}$ of baseband data with ASP (Section 2). Using DSPSR (van Straten \& Bailes 2011), each of the four observations was coherently dedispersed, folded onto 256 phase bins using the ephemeris resulting from the timing campaign (Table 3), and split into the maximum possible number of channels of 1024 over $4 \mathrm{MHz}$, for a velocity resolution of $0.83 \mathrm{~km} \mathrm{~s}^{-1}$. The long Fourier transforms needed for coherent dedispersion obviated further windowing functions.

The two, main and interpulse, on-regions were defined as the series of phase bins with $(\mathrm{S} / \mathrm{N})>3$. The off-pulse regions are the two stretches in between, minus 10 bin margins. Within the off-pulse region, the spectra were directly averaged over all bins to produce the off-pulse spectrum, using PSRCHIVE (van Straten et al. 2012). For the on-pulse spectrum, the spectra were averaged while weighting by the square of the pulsar $\mathrm{S} / \mathrm{N}$ in the concerning on-pulse bin (following Weisberg et al. 2008). For each observation, the channel frequencies were barycentered and converted to velocities relative to the Local Standard of Rest.

The spectra from the four observations were summed, weighted by the square of the pulsar $\mathrm{S} / \mathrm{N}$ in each observation, for both the on- and off-pulse. The intensity scales were calibrated by matching the peak of the off-pulse spectrum to the peak brightness temperature $T_{b}$ as measured in this direction by the Very Large Array Galactic Plane Survey (Stil et al. 2006). Overall "pulsar on" and "pulsar off" spectra were thus produced. Their difference $I(v)$, illustrated in Figure 2, can be attributed to the pulsar minus the absorption. By dividing by $I_{0}$, the broadband unabsorbed strength of the pulsar signal, the relative absorption spectrum for $\mathrm{J} 1906+0746$ was produced, as shown in the right-middle panel of Figure 2. It shows several deep absorption features.

\subsection{Interpretation}

Any absorption features deeper than an optical depth $\tau$ of 0.3 is considered significant (Frail \& Weisberg 1990). Four of these appear, peaking at $4,13,35$, and $63 \mathrm{~km} \mathrm{~s}^{-1}$. To determine the distances to which these velocities correspond, we constructed a Galactic rotation curve (the same as Verbiest et al. 2012) using a distance from the Galactic center to the Sun of $R_{0}=8.5 \mathrm{kpc}$ and a flat rotation of $\Theta_{0}=220 \mathrm{~km} \mathrm{~s}^{-1}$ (Fich et al. 1989).

From this curve (Figure 2, bottom panel), we find that the highest-velocity emission component, at $63 \mathrm{~km} \mathrm{~s}^{-1}$, is emitted near the tangent point. This means the distance lower limit is at or beyond that tangent point at $6.4 \mathrm{kpc}$. By propagating the estimated spread in velocities of $\pm 7 \mathrm{~km} \mathrm{~s}^{-1}$ (Frail \& Weisberg 1990), we determine a distance uncertainty of $0.9 \mathrm{kpc}$.

Any emission peak over $T_{b}=35 \mathrm{~K}$ is thought to be sufficiently bright to allow for an absorption measurement (Weisberg et al. 1979). The upper limit to the distance is thus provided by the farthest-out such peak that is not accompanied by absorption. The first peak to meet these criteria is at $-32 \mathrm{~km} \mathrm{~s}^{-1}$. This means the hard distance upper limit derived using this kinematic distance is $15.9 \pm 0.9 \mathrm{kpc}$, significantly beyond the range allowed from the DM distance.

If the pulsar resides just at the tangent point, few features are expected in the absorption spectrum, as there is only a single chance for absorption at a given velocity. If, however, $\mathrm{J} 1906+0746$ is far beyond the tangent point, there are two chances for absorption at each velocity. The absorption spectrum of nearby pulsar B1904+06 shown in Clifton et al. (1988), for example, is much more crowded than that of J1906+0746. Overall, this suggests that the actual distance to J1906+0746 is close to the lower limit.

Verbiest et al. (2012) present a sophisticated likelihood analysis of such measured limits to produce an overall distance estimate that takes selection effects such as the discovery luminosity bias into account. Using this method, ${ }^{18}$ we combine our upper and lower limits with the discovery mean flux density of $0.55 \mathrm{mJy}$ (Lorimer et al. 2006), and obtain an overall distance estimate of $7.4_{-1.4}^{+2.5} \mathrm{kpc}$. That estimate is larger than the previous, DM-derived distance of $5.40_{-0.60}^{+0.56} \mathrm{kpc}$.

\section{PROFILE EVOLUTION}

The profile of J1906+0746 changes drastically over a timescale of years. The prominent interpulse in the 2004

\footnotetext{
18 http://psrpop.phys.wvu.edu/LKbias
} 


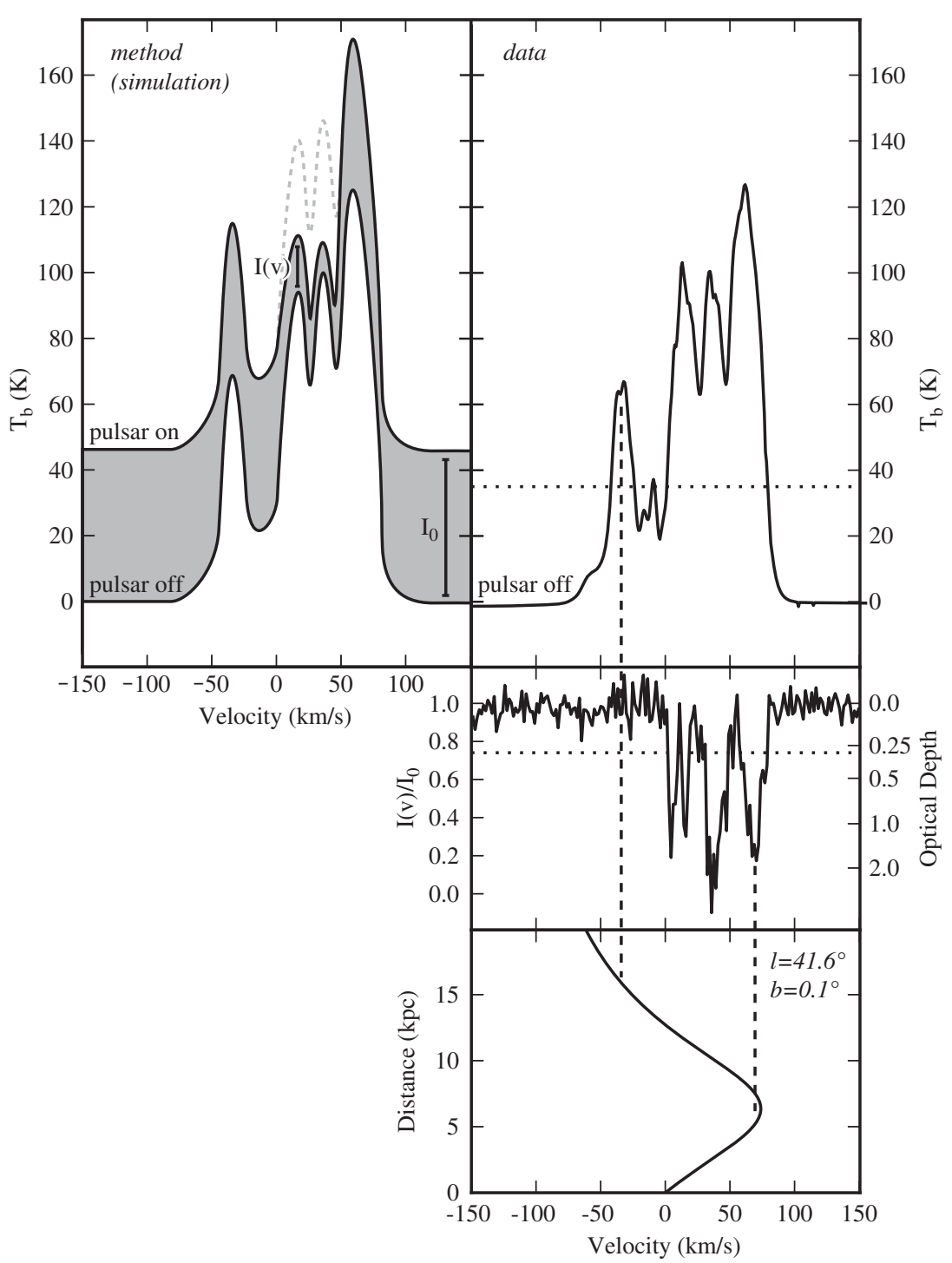

Figure 2. Simulated (left) and measured (right) $\mathrm{H}$ I absorption data. Left: illustration of the subtraction and scaling methods used to define the absorption spectrum $I(v) / I_{0}$. A smoothed version of the measured "pulsar-off" spectrum is shown as the bottom solid curve. The top, gray, dashed curve is based on the measured "pulsar-on" spectrum but the increase in intensity over the pulsar-off spectrum is amplified 100 times for clarity, and shows the expected on-pulse spectrum if there were no absorption and $I(v)=I_{0}$. In the middle, solid, gray-filled curve, which then defines the absorption spectrum $I(v) / I_{0}$, absorption of the two features at 13 and $35 \mathrm{~km} \mathrm{~s}^{-1}$ is simulated. Right: the measured H I spectrum. In the top panel, the off-pulse H I emission spectrum is shown. The $35 \mathrm{~K}$ criterion (Section 3.2 ) is indicated by the dashed horizontal line. The middle panel contains the absorption spectrum $I(v) / I_{0}$. The optical depth, derived from $e^{-\tau}=I(v) / I_{0}$, is noted with a dashed line at $\tau=0.3$. In the bottom panel, the Galactic rotation curve for this line of sight is plotted. The features in the absorption spectrum span the velocity range up to the tangent point. The derived lower and upper bounds to the distance are each marked with a vertical dashed line.

discovery pulse profile was absent from the 1998 Parkes archive profile (Figure 1). On the shortest timescales, our observations range from single-pulse data to $10,30,60$, and 120 s integrations. From visual inspection, we have detected no mode changes on either of these timescales, or in the hours to weeks between observations.

We attribute the profile evolution to geodetic precession of the pulsar's spin angular momentum vector about its total orbital angular momentum vector. In general relativity (GR), the precession rate (e.g., Barker \& O'Connell 1975) is

$$
\Omega_{\text {geod }}=T_{\odot}^{2 / 3}\left(\frac{2 \pi}{P_{b}}\right)^{5 / 3} \frac{1}{1-e^{2}} \frac{m_{2}\left(4 m_{1}+3 m_{2}\right)}{2\left(m_{1}+m_{2}\right)^{4 / 3}}
$$

where $T_{\odot}=G M_{\odot} / c^{3}=4.925490947 \mu$ s is the solar mass expressed in time units; $m_{1}$ and $m_{2}$ are the pulsar and companion masses, respectively, in solar masses; $P_{b}$ is the orbital period, and $e$ is the eccentricity. Using the timing solution and masses presented later, in Table 3, we find a predicted geodetic precession period of $\sim 165 \mathrm{yr}$, which equals a rate of 2.2 degrees per year. Over the 2005-2009 baseline described here, we thus expect a shift in geometry of roughly $10^{\circ}$. The geometry has changed even more significantly — by roughly $20^{\circ}$ - since the 1998 archival Parkes observation, qualitatively consistent with the significantly different pulse shape then.

The secular profile changes observed in J1906+0746 offer an exciting opportunity to study geodetic precession; but the changing profile shape poses a problem in determining precise pulse TOAs. To ensure that the fiducial points of all profiles are consistent, and limit introduction of further timing noise, we used a series of Gaussian standard profiles developed from the well-modeled epochs of ASP, GASP, and WAPP data, and next aligned these Gaussian templates as described below. 
Table 3

Timing Parameters for J1906+0746

\begin{tabular}{|c|c|c|}
\hline Measured Parameter & DD Value & DDGR Value \\
\hline Right ascension, $\alpha$ (J2000.0) & 19:06:48.86(4) & 19:06:48.86(4) \\
\hline Declination, $\delta(\mathrm{J} 2000.0)$ & $07: 46: 25.9(7)$ & $07: 46: 25.9(7)$ \\
\hline Spin Period, $P(\mathrm{~s})$ & $0.14407315538(5)$ & $0.14407315538(5)$ \\
\hline Pulse frequency, $v\left(\mathrm{~s}^{-1}\right)$ & $6.940918295(2)$ & 6.940918295(2) \\
\hline First derivative of pulse frequency, $\dot{v}\left(\mathrm{~s}^{-2}\right)\left(\times 10^{-13}\right)$ & $-9.7643(9)$ & $-9.7642(9)$ \\
\hline Second derivative of pulse frequency, $\ddot{v}\left(\mathrm{~s}^{-3}\right)\left(\times 10^{-23}\right)$ & $5.0(7)$ & $4.9(7)$ \\
\hline Third derivative of pulse frequency $\left(\mathrm{s}^{-4}\right)\left(\times 10^{-30}\right)$ & $-1.8(3)$ & $-1.8(3)$ \\
\hline Fourth derivative of pulse frequency $\left(\mathrm{s}^{-5}\right)\left(\times 10^{-37}\right)$ & $-1.7(2)$ & $-1.7(2)$ \\
\hline Epoch (MJD) & 54289.000001 & 54289.000001 \\
\hline Dispersion measure, $\mathrm{DM}\left(\mathrm{cm}^{-3} \mathrm{pc}\right)$ & $217.7508(4)$ & $217.7508(4)$ \\
\hline Ephemeris & \multicolumn{2}{|c|}{ DE405 } \\
\hline Clock & \multicolumn{2}{|c|}{ TT(BIPM) } \\
\hline Orbital period, $P_{b}$ (days) & $0.16599304683(11)$ & $0.16599304686(11)$ \\
\hline Projected semimajor axis, $x$ (lt s) & $1.4199620(18)$ & $1.4199506(18)$ \\
\hline Orbital eccentricity, $e$ & $0.0853028(6)$ & $0.0852996(6)$ \\
\hline Epoch of periastron, $T_{0}(\mathrm{MJD})$ & $54288.9298810(2)$ & $54288.9298808(2)$ \\
\hline Longitude of periastron, $\omega$ (degrees) & $76.3320(6)$ & $76.3317(6)$ \\
\hline Rate of periastron advance, $\dot{\omega}$ (degrees/yr) & $7.5841(5)$ & \\
\hline Time dilation and gravitational redshift parameter, $\gamma$ & $0.000470(5)$ & \\
\hline Orbital period derivative, $\dot{P}_{b}\left(\times 10^{-12}\right)$ & $-0.56(3)$ & \\
\hline Excess orbital period derivative, $\dot{P}_{b}\left(\times 10^{-12}\right)$ & & $0.03(3)$ \\
\hline Total mass, $M_{\text {total }}\left(M_{\odot}\right)$ & & $2.6134(3)$ \\
\hline Companion mass, $m_{2}\left(M_{\odot}\right)$ & & $1.322(11)$ \\
\hline Derived Parameter & DD Value & DDGR Value \\
\hline Pulsar mass, $m_{1}\left(M_{\odot}\right)$ & & $1.291(11)$ \\
\hline Rate of periastron advance, $\dot{\omega}$ (degrees/yr) & & $7.5844(5)$ \\
\hline Time dilation and gravitational redshift parameter, $\gamma$ & & $0.000470(5)$ \\
\hline Orbital period derivative, $\dot{P}_{b}\left(\times 10^{-12}\right)$ & & $-0.56498(15)$ \\
\hline Inclination angle, $i$ (degrees) & & $43.7(5)$ \\
\hline Galactic latitude, $l$ (degrees) & 41.5982 & \\
\hline Galactic longitude, $b$ (degrees) & 0.1470 & \\
\hline Mass function, $f_{\text {mass }}$ & $0.1115662(2)$ & $0.1115636(4)$ \\
\hline Characteristic age $\tau_{c}=P / 2 \dot{P}(\mathrm{kyr})$ & 112.6 & 112.6 \\
\hline Surface magnetic field, $B_{S}=3.2 \times 10^{19}(P \dot{P})^{1 / 2}\left(10^{12} \mathrm{G}\right)$ & 1.73 & 1.73 \\
\hline DM-derived distance to pulsar, $d_{\mathrm{DM}}(\mathrm{kpc})$ & $5.40_{-0.60}^{+0.56}$ & $5.40_{-0.60}^{+0.56}$ \\
\hline H I-derived distance to pulsar, $d_{\mathrm{H}_{\mathrm{I}}}(\mathrm{kpc})$ & & \\
\hline
\end{tabular}

Notes. The columns contain the parameters measured and derived using, on the left, the DD (Damour \& Deruelle 1986) model-independent timing model; on the right, the DDGR (Taylor 1987) timing model, which assumes general relativity to be the correct theory of gravity. The DD model measures $\dot{\omega}, \gamma$, and $\dot{P}_{b}$, which can each be used to put constraints on the masses of the pulsar and companion. The DDGR model measures the total mass $M_{\text {total }}$ and the companion mass $m_{2}$ directly, and the post-Keplerian parameters can be derived from the values of the masses. The errors on these DDGR parameters $i, \dot{\omega}, \gamma$, and $\dot{P}_{b}$ were derived through a Markov Chain Monte Carlo analysis based on the errors of $M_{\text {total }}$ and $m_{2}$. Other errors reported here are TEMPO $2 \sigma$ values. The DM-derived distance to the pulsar was estimated using the NE2001 model (Cordes \& Lazio 2002).

Using BFIT (Kramer et al. 1994), we fit sets of up to three (as necessary) Gaussians to both the pulse and interpulse in the summed profile of each epoch (illustrated for MJD 54390 in Figure 3). This approach was previously used to determine the geometry of the PSR B1913+16 system (Kramer 1998). We next identified a stable component, and used this component as our timing fiducial point. We found that the smoothest alignment was achieved by keeping the phase of the initially tallest component ("component A") constant. Figure 3 shows this approach for a subset of the ASP and GASP summed profiles. After modified Julian day (MJD) 54700 we could no longer reliably identify component $\mathrm{A}$, and so instead aligned the tallest component for those epochs (component B), and introduced a fiducial point phase shift based on the transition profile in which components A and B were both identifiable (see Figure 3). The full collection of Gaussian-modeled profiles is shown in Figure 4. This method of aligning the profiles produced fairly monotonic behavior in the phase of the interpulse, as can be seen by the gradual widening between the interpulse peaks and the fixed, vertical dashed line in Figure 4. After this alignment, these profiles were used as the standards for highprecision timing.

\subsection{Profile Flux Variations}

Throughout the period covered by this study, the profile evolution of J1906+0746 was accompanied by a steady decrease in the pulsar mean flux density (Figure 1). Independently processed, well calibrated, ASP and GASP data produced mean flux density estimates that are consistent, falling from $0.8 \mathrm{mJy}$ in 2006 to $0.2 \mathrm{mJy}$ in 2009 (Figure 4.6 in Kasian 2012). 

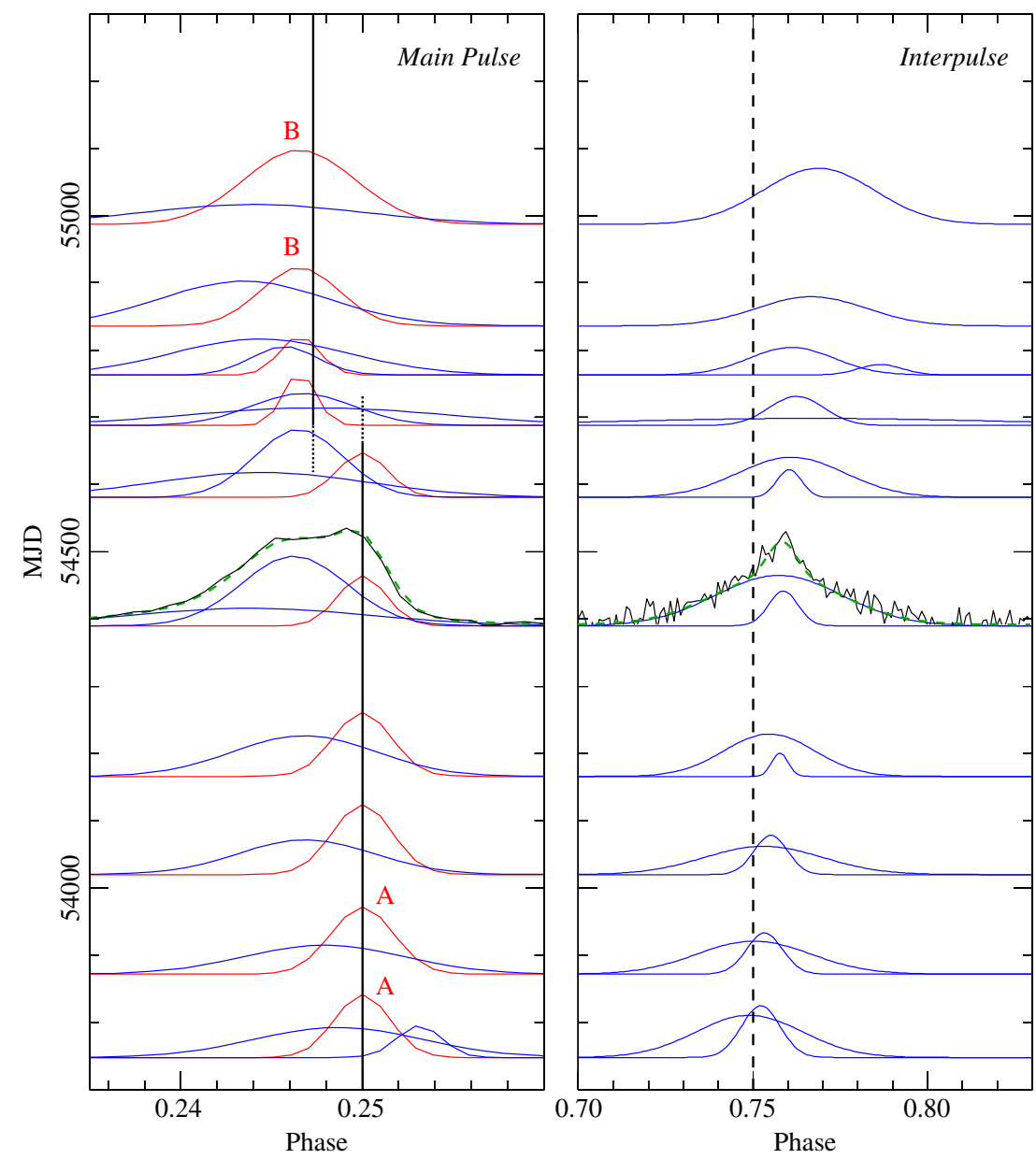

Figure 3. Illustration of the alignment based on Gaussian components, for GASP and ASP profiles. Shown are the main pulse (left) and interpulse (right, flux scale increased seven times). The vertical line at phase 0.25 marks the location of the fiducial point. The vertical line at phase 0.75 can be used to trace the changing location of the interpulse, over time. The red lines represent the Gaussian component A or B that was used for the alignment. The blue lines are the other Gaussian components. For MJD 54390 the data (black line) and total profile model (dashed green line) are also shown.

\section{TIMING}

\subsection{Times of Arrival}

TOAs were created for the profiles resulting from the iterative data reduction process described in Section 2.1. For the WAPP Arecibo data, we created separate profiles for each epoch and each frequency. The Gaussian-derived templates arising from ASP, GASP, and the three WAPPs are shown in Figure 4. As noted, timing standard profiles are commonly created with their main peak as the fiducial point, at zero phase; here, in contrast, we preserved the alignment of the Gaussian-derived templates, as is clear from Figure 4. For Arecibo, GBT, and Nançay timing, we used the evolving Gaussian templates described above (Section 4). At Arecibo, there were unexplained offsets between the three WAPP machines on MJD 53810, so that day is left out of the analysis. For the higher-cadence Nançay data, interpolation of the produced profiles provided a smooth evolution of the components. For Jodrell and Westerbork timing, static standard profiles were created from bright, aligned subsets of their respective observation campaigns.

\subsection{Timing Solution for J1906+0746}

The complete set of 28,000 TOAs was fit for the parameters describing the state and evolution of both the individual pulsar and the binary system. This initial fitting was performed using the TEMPO2 (Hobbs et al. 2006) package. Using the data from Arecibo, GBT, Jodrell, Nançay, and Westerbork, we produced a phase-connected solution over the entire period, effectively accounting for every one of the $10^{9}$ rotations over the 2005-2009 time span. J1906+0746 has a large amount of timing noise, however, that is difficult to decouple from the orbital parameters. We first attempted to subtract this noise using FITWAVES (Hobbs et al. 2004), but better noise removal was achieved by modeling the pulsar rotation frequency as a 10th order polynomial in time, the highest degree of complexity currently implemented in TEMPO2. The residuals of that timing solution are shown in Figure 5.

Even this last solution, however, shows large variations (of $420 \mu \mathrm{s} \mathrm{rms}$ ) due to remaining timing noise or unmodeled profile variations. To determine the system parameters with the highest precision, we thus chose to include only the timing data that used the evolving timing profiles, from Arecibo, GBT, and Nançay (Section 5.1). (The data from Jodrell and Westerbork were produced with static profiles and were well suited for inclusion in the long-term high-cadence timing solution, but did not help the orbital timing solution.) In an attempt to limit the effects of timing noise and home in on the best set of orbital parameters, we fit for an arbitrary offset around every set of TOAs derived from a single observation as well as individual-day DM values on days with multi-WAPP Arecibo observations (see Section 6.1). The 

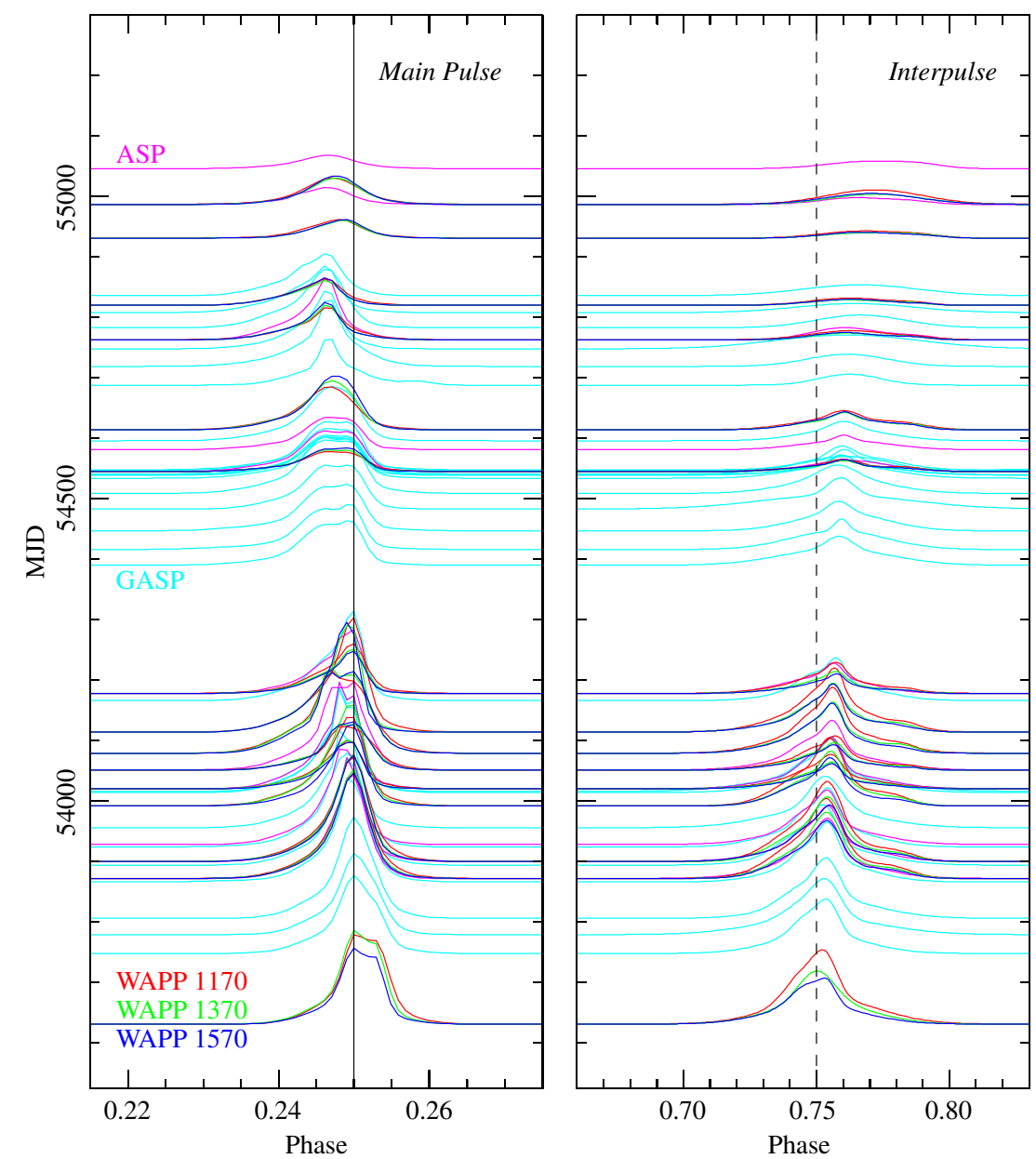

Figure 4. Resulting fits to the pulse and interpulse of J1906+0746, based on data taken with the WAPPs (red: $1170 \mathrm{MHz}$, green: $1370 \mathrm{MHz}$, blue: $1570 \mathrm{MHz}$ ASP (magenta) and GASP (cyan). The vertical axis spans from 2005 July to 2009 August. The interpulse flux is magnified by a factor of 10 relative to the main pulse. The vertical line for the main pulse (left) illustrates the chosen alignment. A vertical line in the interpulse panel (right) illuminates the interpulse phase shift.

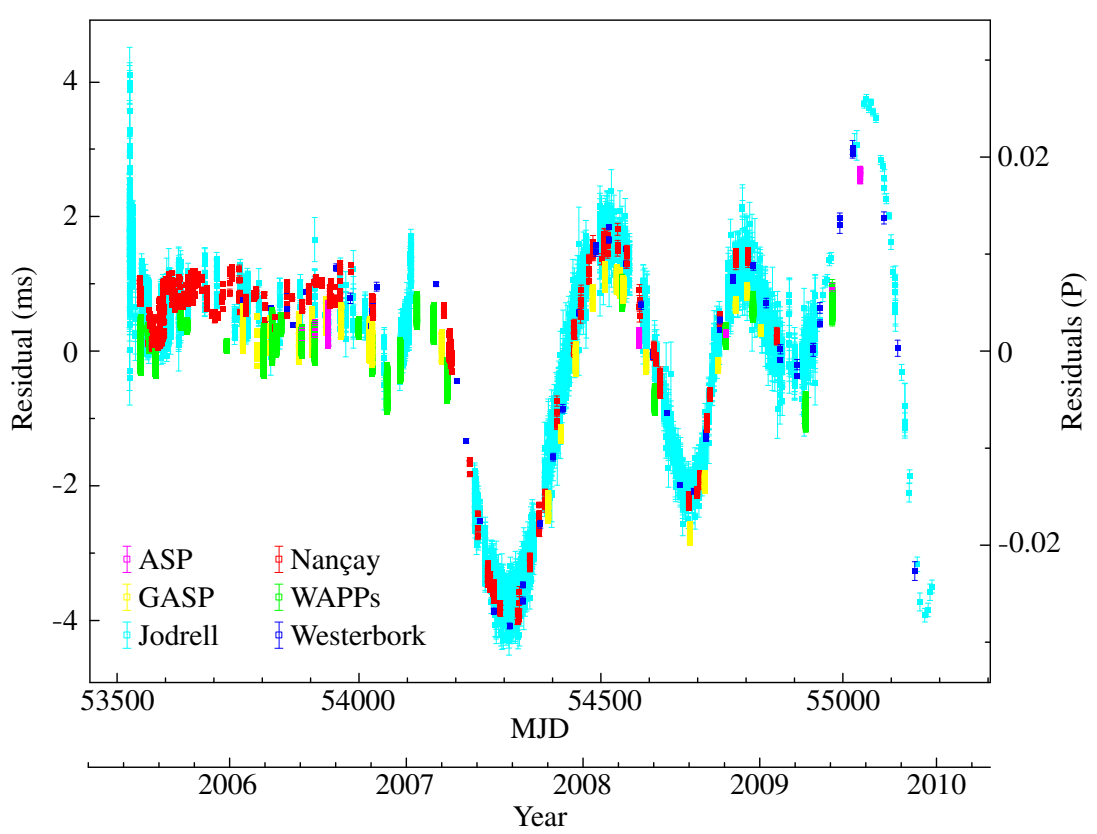

Figure 5. Residuals plotted vs. MJD and Year for the entire data set. Shown are the residuals after using the timing solution that included a 10th order polynomial in time. Also fitted were offsets between the various observatories, between pre- and post-54100 Jodrell data, and between PuMa and PuMaII. Data from ASP are magenta, GASP is yellow, Jodrell is cyan, Nançay is red, the combined WAPP data are shown in green, and Westerbork is blue. 


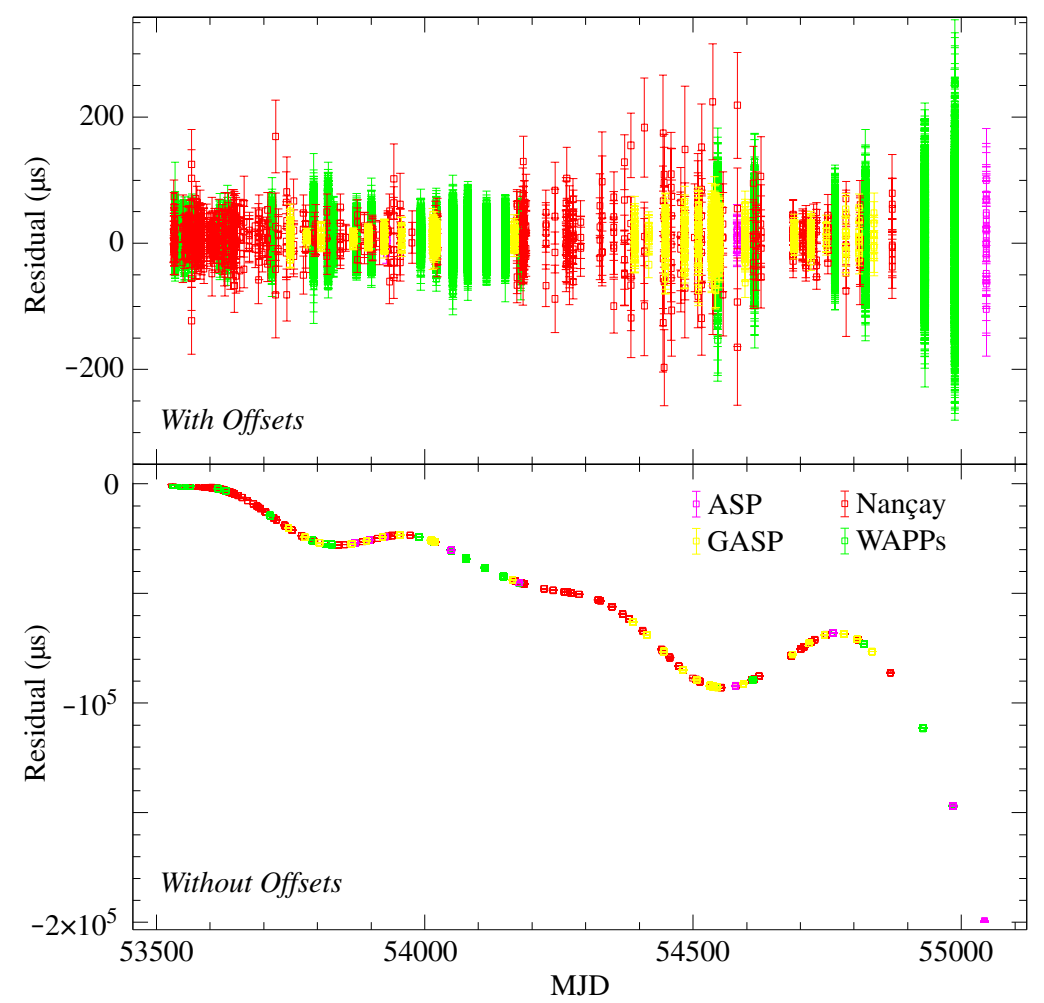

Figure 6. Residuals are plotted vs. MJD for the best fit. The top panel shows the residuals for the solution that includes jumps between the 118 individual epochs. Those jumps remove the long-term timing noise trend that is clearly visible in the bottom panel. There we show the same phase-connected timing solution, but without any jumps between epochs. Green residuals represent WAPP Arecibo data, magenta represents ASP Arecibo, yellow is GASP Green Bank, and red is Nançay data.

TEMPO ${ }^{19}$ code allows up to a 20 th order polynomial in frequency, and we tested fitting each of these orders while simultaneously fitting the offsets, DM values, position and spin, and binary parameters. The fit that allowed for a fourth-order frequency polynomial produced the lowest reduced $\chi^{2}$ and was adopted as our preferred timing solution.

The final, best timing solution is presented in Table 3, and the resulting residuals $(17 \mu \mathrm{s} \mathrm{rms})$ are shown in the top panel of Figure 6. We re-weighted our data so that the reduced $\chi^{2}$ of the fit was equal to one for each data set, and overall. Following common use, all reported uncertainties are twice the values produced by TEMPO after this re-weighting.

\subsection{Measurement of Post-Keplerian Parameters}

Our timing solution presented in Table 3 includes several post-Keplerian parameters. We describe these in some more detail below.

\subsubsection{Measuring $\gamma$, the Gravitational Redshift}

We tested how our measurement of $\gamma$ depends on the current stage of the periastron precession cycle, which affects the viewing geometry. We simulated TOAs over various points spanning one whole cycle, as was previously shown for PSR B1913+16 and B1534+12, in Damour \& Taylor (1992, Figure 5, top line). We find that our set of TOAs, collected while $\omega$ moved from $\sim 68^{\circ}$ to $78^{\circ}$, corresponds to relatively low theoretical fractional uncertainties, and we are moving toward even better measurability as $\omega$ increases (Kasian 2012).

\footnotetext{
$19 \mathrm{http}: / /$ tempo.sourceforge.net/
}

\subsubsection{Measuring $\dot{P}_{b}$, the Orbital Period Derivative}

The observed value of $\dot{P}_{b}$ needs to be corrected for two effects before it can be compared to the value predicted by GR and used to constrain the system masses. These are, first, the different Galactic acceleration felt by the pulsar and by Earth; and second, the Shklovskii effect (Shklovskii 1970), which incorporates the Doppler effect caused by the pulsar proper motion into the measured $\dot{P}_{b}$ value. We have no measurement of proper motion for this pulsar, but we can estimate the Galactic contribution and calculate the limit on the Shklovskii contribution and the system proper motion. If that limit is in line with the measured proper motions of similar systems, $\dot{P}_{b}$ can be used to constrain the binary system. Following Damour \& Taylor (1991) and Nice \& Taylor (1995), we write the observed orbital period decay $\dot{P}_{b}^{\text {obs }}$ as

$$
\dot{P}_{b}^{\text {obs }}=\dot{P}_{b}^{\text {int }}+\dot{P}_{b}^{\text {Gal }}+\dot{P}_{b}^{\text {Shk }},
$$

where the Galactic contribution $\dot{P}^{\text {Gal }}$ and Skhlovskii term $\dot{P}_{b}^{\text {Shk }}$ add to the intrinsic decay $\dot{P}_{b}^{\text {int }}$. If we assume that $\dot{P}_{b}^{\text {int }}$ is equal to $\dot{P}_{b}^{\mathrm{GR}}$, the value determined by GR, we can rewrite Equation (2) to isolate the Shklovskii contribution, scaled to the binary orbit:

$$
\left(\frac{\dot{P}_{b}}{P_{b}}\right)^{\mathrm{Shk}}=\left(\frac{\dot{P}_{b}}{P_{b}}\right)^{\mathrm{obs}}-\left(\frac{\dot{P}_{b}}{P_{b}}\right)^{\mathrm{GR}}-\left(\frac{\dot{P}_{b}}{P_{b}}\right)^{\mathrm{Gal}} .
$$

On the right-hand side of Equation (3), each of the terms can be estimated or calculated. First, our best-fit value of the orbital decay $\dot{P}_{b}^{\text {obs }}$ is $-0.56(3) \times 10^{-12}$ (Table 3 ).

Second, the value predicted by GR and computed from fitting the DDGR model (Damour \& Deruelle 1986; Taylor \& Weisberg 1989 ) to our data $\dot{P}_{b}^{\mathrm{GR}}$ is $-0.56498(15) \times 10^{-12}$ (Table 3 ). 


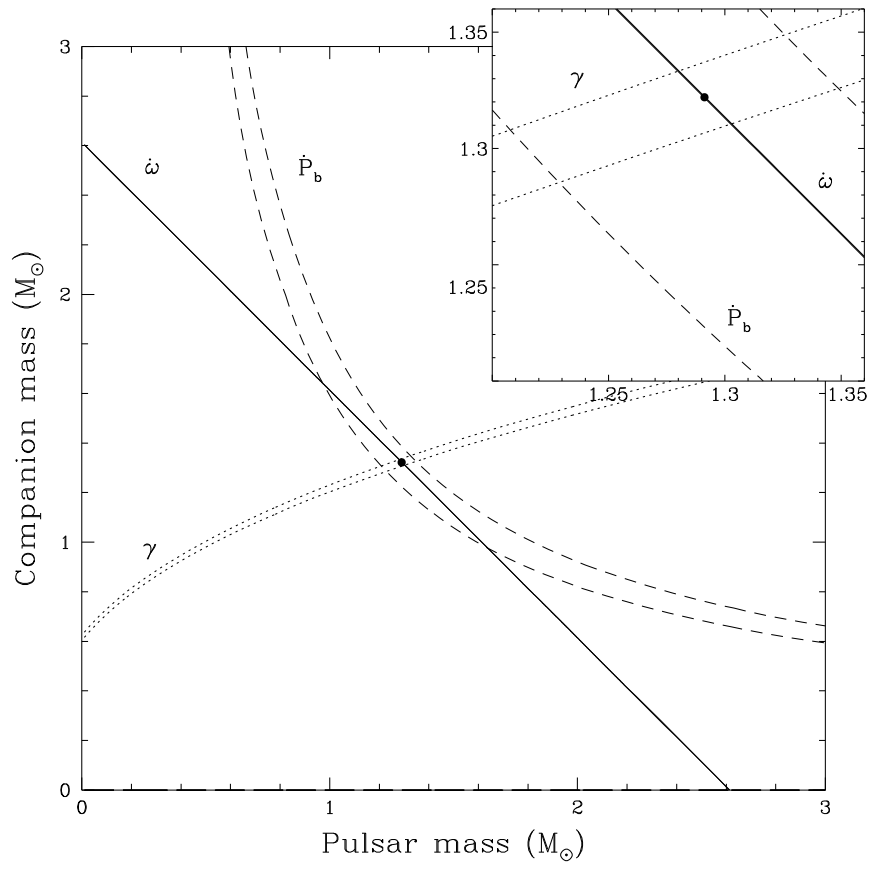

Figure 7. Mass-mass diagram for the ephemeris presented in Table 3. The lines represent the values of $m_{1}$ and $m_{2}$ allowed by the three measured DD postKeplerian parameters: $\dot{\omega}$ (the solid line), $\gamma$ (the dotted lines), and $\dot{P}_{b}$ (the dashed lines). The dot indicates the best-fit value for $m_{1}$ and $m_{2}$.

The third term, the Galactic contribution, can be written as $\left(\dot{P}_{b} / P_{b}\right)^{\mathrm{Gal}}=\boldsymbol{a} \cdot \hat{n} / c$, where $\boldsymbol{a}$ is the differential acceleration in the field of the galaxy and $\hat{n}$ is the unit vector along our line of sight to the pulsar. The components parallel and perpendicular to the Galactic plane that make up this term can be calculated (Nice \& Taylor 1995, Equations (3)-(5)). Using (again) $\Theta_{0}=220 \mathrm{~km} \mathrm{~s}^{-1}$ and $R_{0}=8.5 \mathrm{kpc}$, plus the pulsar coordinates and $\mathrm{H}$ I-absorption distance from Table 3 , we find $(\boldsymbol{a} \cdot \hat{n} / c)_{\|}=(6.3 \pm 2.5) \times 10^{-19} \mathrm{~s}^{-1}$ and $(\boldsymbol{a} \cdot \hat{n} / c)_{\perp}=$ $(4.5 \pm 1.1) \times 10^{-23} \mathrm{~s}^{-1}$. These translate to a Galactic correction $\dot{P}_{b}^{\text {Gal }}=0.009(4) \times 10^{-12}$. Given the large distance uncertainty, this value does not significantly change when using the more recent Reid et al. (2014) Galactic kinematics. Combined, the three terms limit the Shklovskii contribution to be essentially zero, $\dot{P}_{b}^{\text {Shk }}=P_{b} \mu^{2} d_{\mathrm{HI}} / c<0.03 \times 10^{-12}$ (at $95 \%$ confidence level), where $\mu$ and $d_{\mathrm{HI}}$ are the total proper motion and the distance of the pulsar, respectively. The error ranges on $P_{b}$ and $d_{\mathrm{H}_{\mathrm{I}}}$ allow for a proper motion of $<9$ mas/yr or transverse velocity of $v=\mu d_{\mathrm{H} \text { I }}<400 \mathrm{~km} \mathrm{~s}^{-1}$ (95\% CL). This easily encompasses the range of published system velocities for other relativistic pulsars (e.g., Hobbs et al. 2005).

Our results thus imply that the orbital period decay $\dot{P}_{b}$ for J1906+0746 is consistent with the value predicted by GR.

\subsection{Mass Measurements}

Having obtained reasonable estimates of the advance of periastron $\dot{\omega}$, the gravitational redshift/time dilation parameter $\gamma$, and the orbital decay $\dot{P}_{b}$ for J1906+0746, we use these three parameters to place constraints on the masses of the pulsar $\left(m_{1}\right)$ and companion $\left(m_{2}\right)$.

If we use the dependence of the post-Keplerian parameters on the masses, as defined in general relativity (see, e.g., Taylor \& Weisberg 1989), each parameter constrains the allowed $\left(m_{1}, m_{2}\right)$ pairs. The intersection of the allowed regions in $m_{1}-m_{2}$ parameter space represents the most likely values of the

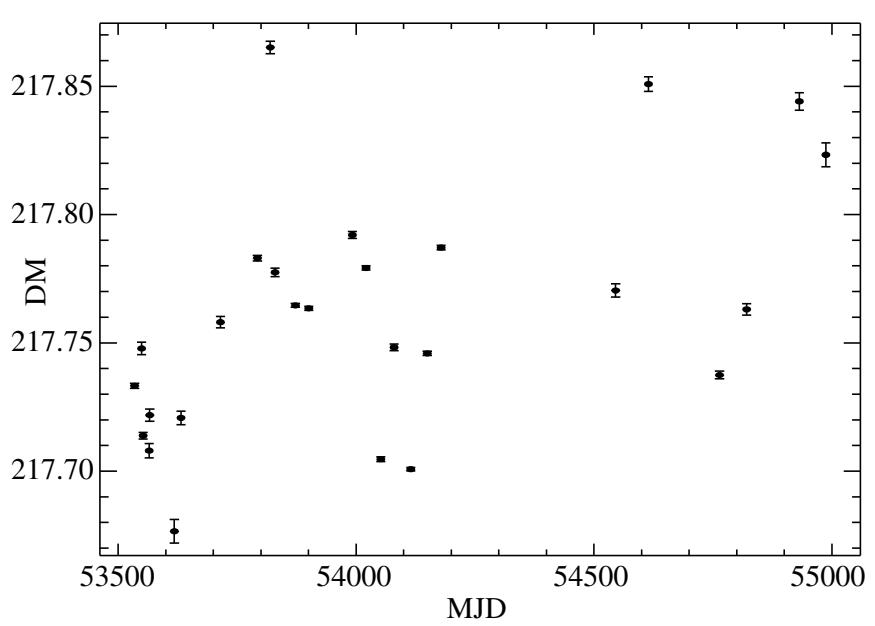

Figure 8. Dispersion measure variations vs. time, for all WAPP-data epochs, fit simultaneously with the rest of the timing model.

pulsar and companion masses. The mass-mass diagram for our timing solution of J1906+0746 is shown in Figure 7. The three measured post-Keplerian parameters provide consistent values of the pulsar and companion masses. Using the DDGR (Damour \& Deruelle 1986, Taylor \& Weisberg 1989) binary model, we finally conclude that the pulsar mass $m_{1}=1.291(11) M_{\odot}$ and the companion mass $m_{2}=1.322(11) M_{\odot}$. That pair of masses is marked in Figure 7, and indeed falls within the overlap of the constraints from the post-Keplerian parameters.

These masses differ from the initial estimates reported in Kasian (2008), at $m_{p}=1.248(18) M_{\odot}$ and $m_{c}=1.365(18) M_{\odot}$ for the pulsar and companion, respectively. This change can be explained by the longer data span used here, and by the improved method of eliminating the strong long-term timing noise.

\section{DISPERSION MEASURE VARIATIONS}

We next investigate changes in the DM, either as long-term evolution or as trends that could recur every orbit. To estimate such variations within our data span, we used the WAPP data, with its superior wide bandwidth and 512 channel spectral information.

\subsection{Secular DM Variation}

For each epoch of WAPP data with three WAPPs, we used TEMPO to fit for the DM using only TOAs from that day. We accomplished this by using the "DMX" model within TEMPO, fitting the individual-day DM values simultaneously with the rest of the timing solution. This can bring to light intrinsic DM variations, but it can also absorb other frequency dependent effects. Note that the ASP data at these epochs were still fit with surrounding offsets because they did not, in general, agree with the WAPP DM values. This is presumably due to the fact that the ASP profiles were obtained with coherent dedispersion but also folded in real-time with some unavoidable ephemeris smearing.

Generally, the observed DM variations are larger than the error bars. There is, however, no long-term behavior that could be included in the timing analysis (Figure 8). The short-term variations observed in the DM values are most likely induced by other TOA changes that systemically depend on frequency; these could be profile evolution that varies with frequency, potentially amplified when scintillation affects the relative contributions of different parts of the band. 


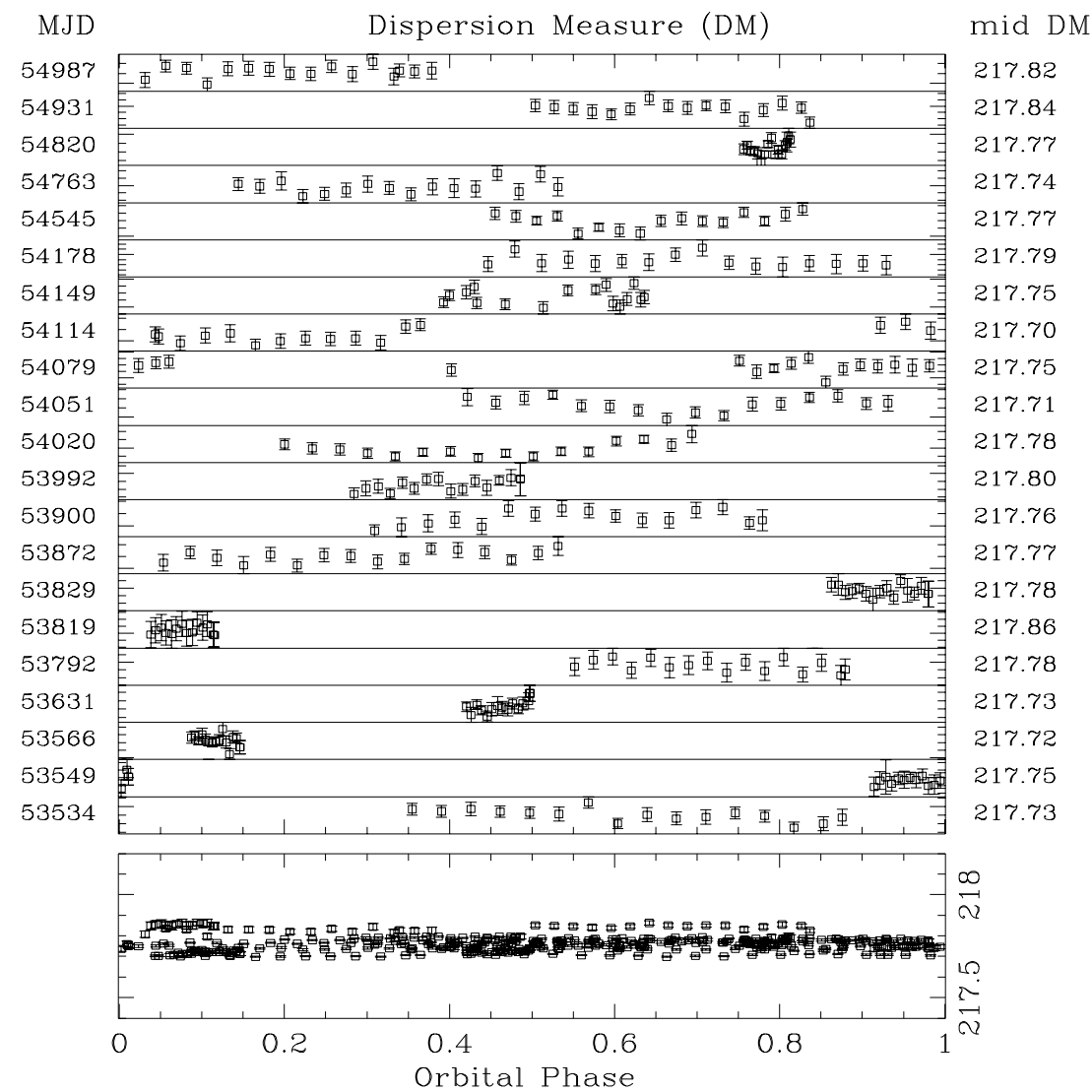

Figure 9. DM vs. orbital phase for all epochs where good WAPP data were available for J1906+0746, folded on the Kasian (2012) ephemeris. The bottom plot shows the DM over orbital phase for all of the epochs together.

\subsection{Orbital DM Variation}

The DM may vary with orbital phase if the pulsar emission travels through varying plasma densities throughout the binary orbit. Such variations have been seen in several pulsars with non-degenerate companions (e.g., B1957+20, Fruchter et al. 1995; and J2051-0827, Stappers et al. 2001). To distinguish between a NS and a less compact companion, we investigated the behavior of the DM of J1906+0746 over orbital phase.

For each WAPP data epoch, we divided TOAs over 16 bins across the $\sim 2 \mathrm{hr}$ Arecibo observation. We calculated the bestfit DM for each of the 16 bins individually, keeping other timing parameters fixed at the best-fit values (Table 3 ). We then investigate the DM versus orbital phase for each epoch, as plotted in Figure 9. Although variations at individual epochs are somewhat significant within the stated, TEMPO-doubled error bars, there are no compelling overall trends in DM over the course of an orbit, and no evidence for a more extended companion.

\section{ORBITAL ABERRATION}

We have so far attributed the observed profile changes to geodetic precession, an effect seen only in strong gravitational fields. It is, however, also possible that the special relativistic effect of aberration contributes to the observed profile changes. Aberration on an orbital timescale arises from the relativistic velocities with which the pulsar and companion travel in their orbit (Damour \& Taylor 1992; Rafikov \& Lai 2006), and was measured in the double neutron star (DNS) B1534+12 (Stairs et al. 2004; Fonseca et al. 2014). As the velocity at periastron of $\mathrm{J} 1906+0746, v_{p} \sim 0.001 c$, is similar to that of $\mathrm{B} 1534+12$
(Equation (8.35); Lorimer \& Kramer 2005), one might expect to detect aberration. A longitudinal delay will shift the pulse profile in phase, while keeping the shape of the pulse intact; meanwhile, a latitudinal delay shifts the observed emission angle with respect to the pulsar spin axis (Rafikov \& Lai 2006). That latter change in our line of sight could produce measurable profile changes over the course of a binary orbit.

If detected, we can combine the profile changes due to orbital aberration with the secular changes due to geodetic precession to put limits on the geometry of the system (Stairs et al. 2004). Fitting the polarimetry data for J1906+0746 to the classical rotating vector model resulted in the following measured angles (Desvignes 2009): the current angle between the spin and magnetic axes $\alpha=80_{-6}^{\circ+4}$; the geodetic precession phase $\Phi_{\text {SO }}^{0}=109_{-79}^{\circ+51}$; and the precession cone opening angle (or misalignment angle) $\delta=110_{-55}^{\circ+21}$. A detection of orbital aberration could constrain further angles and would allow for a measurement of the geodetic precession period independent of the profile beam model.

\subsection{Observations}

The detection of profile changes on an orbital timescale, against a backdrop of steady profile evolution from geodetic precession, requires several complete orbits of coverage collected over a relatively short time span. We thus collected GBT GASP data during two separate, high-cadence campaigns-over four days between 2006 October 4 and 12, and over 14 days between 2008 March 9 and 23. These campaigns (compare to Section 2) are each much shorter than the $\sim 165$ yr geodetic 

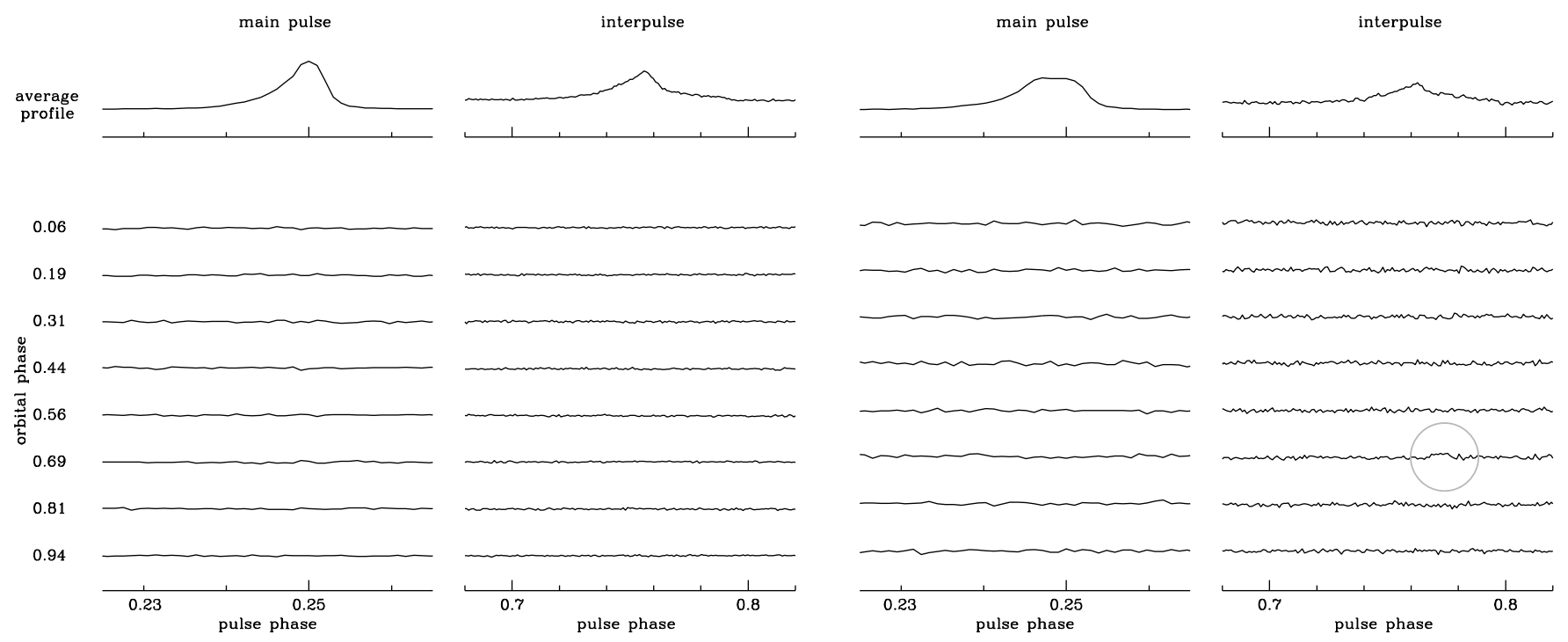

Figure 10. Pulse and interpulse average profile of J1906+0746 (top row), and the difference between these total averages, and the average for eight orbital phase bins (bottom rows). Data were taken over four days in 2006 October (left) and three days in 2008 March (right). The interpulse flux has been magnified eight-fold relative to the main pulse. The top row shows the average profiles per campaign. The subpanels below show the difference of the profile at orbital phase compared to the average.

precession period. The fully steerable GBT allowed tracks of the pulsar for at least one full orbit per day, in both campaigns.

\subsection{Method}

To measure pulse profile changes versus orbit, separately for each campaign, we used the following approach, adapted from Ferdman et al. (2008). We produced time-averaged pulse profiles per five minute intervals, using the ephemeris derived by Kasian (2012). From these, we first created an average profile for the entire campaign. Each five-minute profile was next binned by true anomaly, over eight orbital phases. After scaling all profiles to a uniform height, the bins were summed. We then investigated the differences, shown in Figure 10, between the total profile and each binned profile.

For both the interpulse and the main pulse, we subtracted a linear baseline over their respective data window (the horizontal range in the subplots of Figure 10). We then looked for series of five subsequent time bins that were offset from the mean by more than one standard deviation.

\subsection{Results}

Only one such instance was found, indicated with the gray circle in Figure 10, but no related changes were detected at other orbital phases. Thus, no variations on orbital timescales were detected with this method. The difference profiles, shown in Figure 10, do not show significant other changes over the course of an orbit. From this, we conclude that J1906+0746 has a small aberration amplitude; and that the long-term profile evolution can be used to constrain the emission beam.

\section{NATURE OF THE COMPANION}

From our timing campaign, we find a companion mass $m_{c}=$ $1.322(11) M_{\odot}$ and a pulsar mass $m_{p}=1.291(11) M_{\odot}$. As evident from Table 1, these masses fit the observed collection of DNSs well, and the standard model for DNS evolution, in which the recycled companion is more massive than-or at least comparable to-the young NS. The mass of the companion is, however, also similar to that of the massive WD in the relativistic binary with young pulsar B2303+46 (Table 1). Thus, the masses alone cannot rule out that the companion is a WD.

For some binary pulsars, it is possible to observe the WD companion optically; however, as discussed in Lorimer et al. (2006), this is not a viable option for J1906+0746. As detailed in the next section, we would expect a WD companion to have an age of at least $\sim 1 \mathrm{Myr}$. Now, older and equally distant WDs have been optically detected (B2303+46; van Kerkwijk \& Kulkarni 1999); but for the low Galactic latitude of J1906+0746 ( $b=$ 0.15 ) the modeled extinction $A_{V}$ out to $d_{\mathrm{HI}}=7.4 \mathrm{kpc}$ can range from 4.1-8.4 (models A-S in Amôres \& Lépine 2005). That suggests a $V$ magnitude of at least 29 , too faint to reasonably detect. Optical studies of the companion can therefore not confirm or rule out its nature.

If J1906+0746 is part of a DNS binary, it may be possible to detect the companion as a second radio pulsar-as seen in the double pulsar system, J0737-3039A/B (Lyne et al. 2004). We have, therefore, performed searches for pulsed emission from the companion in the WAPP and Spigot data. A SIGPROC time series, dedispersed at the DM of the known pulsar, was transformed into the companion rest frame for a range of possible projected semimajor axes. We then searched for periodicities in these data, and folded the transformed time series at the candidate periods. No convincing pulsar signals were found. If the companion is a pulsar, it is either beamed away from Earth; or too dim. The minimum flux density $S_{\min }$ that we could have detected was

$$
S_{\min }=\beta \frac{\sigma_{\min }\left[T_{\mathrm{sys}}+T_{\mathrm{sky}}(l, b)\right]}{G \sqrt{n_{p} t_{\mathrm{obs}} \Delta v}} \sqrt{\frac{W_{e}}{P-W_{e}}}
$$

(Dicke 1946; Dewey et al. 1985), where $\sigma_{\min }=8$ is the threshold detection $\mathrm{S} / \mathrm{N}, n_{p}=2$ is the number of summed polarizations, $\Delta v$ is the bandwidth $(600 \mathrm{MHz}$ with the Spigot; $3 \times 100 \mathrm{MHz}$ for the WAPPs), $\beta \sim 1.2$ is the quantization factor for three-level quantization (Lorimer \& Kramer 2005), $G$ is the antenna gain $\left(2.0 \mathrm{~K} \mathrm{Jy}^{-1}\right.$ for the $L$-band receiver at the 
$\mathrm{GBT}^{20}$ and $10 \mathrm{~K} \mathrm{Jy}^{-1}$ for the L-wide receiver at Arecibo, ${ }^{21}$ ) $T_{\text {sys }}$ is the system temperature $(20 \mathrm{~K}$ and $25 \mathrm{~K}$ for the GBT and Arecibo, respectively), $T_{\text {sky }}(l, b)$ is the temperature of the sky at the location of the source (Haslam et al. 1982), $W_{e}$ is the effective pulse width of the pulsar, and $P$ is the pulse period. For an integration time of $t_{\mathrm{obs}} \sim 8 \mathrm{hr}$ with Green Bank, the longest observation we searched, and assuming an effective pulse width of $10 \%$, our flux density limit is $S_{\min } \simeq 19.7 \mu \mathrm{Jy}$. For our $\sim 2 \mathrm{hr}$ observations with Arecibo, we reach $S_{\min } \simeq 19.3 \mu \mathrm{Jy}$. At the Hi-absorption distance of $7.4 \mathrm{kpc}$, any companion pulsar beamed toward us thus has a pseudoluminosity $S_{1400} d^{2}<1.1 \mathrm{mJy} \mathrm{kpc}^{2}$. Comparing this with the $L$-band pseudoluminosities of the recycled pulsars in known DNSs (1.9 mJy kpc ${ }^{2}$ for J0737-3039A, 0.6 mJy kpc ${ }^{2}$ for B1534+12, and $45 \mathrm{mJy} \mathrm{kpc}^{2}$ for B1913+16-Burgay et al. 2006; Deller et al. 2009a; Kramer et al. 1998; Taylor \& Cordes 1993) we conclude that our search would have detected two out of three of these at the $\mathrm{H}_{\mathrm{I}}$ distance of J1906+0746, and thus had sufficient sensitivity to detect the average known recycled pulsar in a DNS if its beam intersected Earth.

If the opening angle between the spin axis of this NS companion and the angular momentum of the orbit is large enough, the putative recycled pulsar will become visible within a geodetic precession timescale (Equation (1)). Continued follow up and searches for pulsations may thus prove that the companion is a NS.

Without a direct optical or radio detection at the moment, the nature of the companion remains best investigated by comparing the J1906+0746 system masses to the collection of known DNS and relativistic WD binaries with precise mass estimates (Table 1). The companion mass $m_{c}=1.322(11) M_{\odot}$ is likely higher than that of the most massive known similar WD, the $1.3_{-0.10}^{+0.10} M_{\odot}$ companion to PSR B2303+46. The companion mass reported does, however, fall well within the mass range of the recycled stars in known DNSs.

\section{IMPLICATIONS AND CONCLUSION}

We presented an updated timing solution for J1906+0746 that allows for the measurement of three post-Keplerian parameters: $\dot{\omega}, \gamma$, and $\dot{P}_{b}$. We measured pulsar and companion masses of $m_{p}=1.291(11) M_{\odot}$ and $m_{c}=1.322(11) M_{\odot}$, respectively, compatible with a NS or possibly a WD companion.

If the binary companion to this young unrecycled pulsar is a WD, it must have formed first. Such systems are observationally rarer by an order of magnitude than NS-WD systems in which the NS is recycled. They also require a different mass transfer history than the average binary pulsar.

The existence of young pulsars in binaries around WDs was predicted by Dewey et al. (1985) and Tutukov \& Yungelson (1993), and was subsequently confirmed by the detections of the 12 day binary B2303+46 (Thorsett et al. 1993; later identified as a NS-WD system by observation of the WD companion; van Kerkwijk \& Kulkarni 1999) and the relativistic binary J1141-6545 (Kaspi et al. 2000; Bhat et al. 2008), where the WD component was optically detected by Antoniadis et al. (2011). These systems do not fit in the traditional spin-up scenario.

An evolutionary channel that can explain this class is outlined in Tauris \& Sennels (2000): the binary progenitor involves a primary star with mass between 5 and $11 M_{\odot}$ and a secondary with initial mass between 3 and $11 M_{\odot}$. The primary evolves

\footnotetext{
20 http://www.gb.nrao.edu/ fghigo/gbtdoc/sens.html

21 http://naic.edu/ astro/RXstatus/Lwide/Lwide.shtml
}

and overflows its Roche lobe, and the secondary accretes a substantial amount of mass during this phase, which lasts $\sim 1$ Myr. At some point after the primary forms a WD, the now-massive secondary evolves and a common envelope is formed for a second, short, mass-transfer phase. The envelope is ejected, and a supernova occurs later, forming the observed young pulsar.

Pulsar J1906+0746 and its possible WD companion would form a binary system of widely different binding energies. In alternative theories of gravity, particularly the Scalar-Tensor theories of gravity (Will 1993; Damour \& Esposito-Farese 1996), such "asymmetric" systems emit dipolar gravitational waves in addition to the quadrupolar emission predicted by general relativity (GR). Such theories can thus be stringently tested by measuring the orbital decay in WD-NS systems (as done for PSR J1141-6545 by Bhat et al. 2008). For symmetric systems like a DNS, which can have striking precision in the measurement of their $\dot{P}_{b}$ (Kramer et al. 2006; Weisberg et al. 2010), only very little dipolar gravitational-wave emission is predicted by alternative theories of gravity.

Thus, for a pulsar-WD system, the closer the agreement of the observed $P_{b}$ with the value predicted by general relativity, the stronger are the constraints on any alternative theories that predict extra gravitational wave emission. Currently, the best limits on such alternatives come from the measurement of the orbital decay of the MSP-WD system PSR J1738+0333 (Freire et al. 2012). The main limitation in the precision of this test is the precision (and accuracy) of the masses of the components of that system, which was derived from optical spectroscopy and is limited, to some extent, by uncertainties in the atmospheric models used (Antoniadis et al. 2012). This is not so much a limitation for PSR J1906+0746, where the masses are known to very good relative precision from the measurement of $\dot{\omega}$ and $\gamma$. Therefore, if the companion of PSR J1906+0746 is indeed a WD, then this system could, in principle, provide a test of alternative theories of gravity that is far superior to any current test. However, for that to happen, three conditions must be fulfilled. First, the companion should be confirmed to be a WD, as Antoniadis et al. (2011) did for the companion of PSR J1141-6545. Second, the parallax and proper motion should be precisely measured to better estimate the kinematic corrections to $\dot{P}_{b}$ (Section 5.3.2). Finally, the measurement of the orbital decay of the system should be improved via timing. The first condition is hard to fulfill because a WD companion is expected to be very faint at optical wavelengths (Section 8). The last two conditions are hard to fulfill at the moment given the fact that the pulsar is becoming very faint in the radio (Section 3 ).

If the companion is a recycled pulsar, there will have been no tidal circularization of the current orbit; nor will there have been enough time for gravitational-wave emission to significantly circularize it since the pulsar is only roughly $\tau_{c}=112.6 \mathrm{kyr}$ old (Table 3). Therefore, the current eccentricity $e=0.0853028(6)$, the lowest of any of the known DNSs, must reflect the state of the orbit after the second supernova. This implies a small supernova kick to the newborn young pulsar J1906+0746, well within our upper limit on the system velocity.

For the general population of relativistic binaries, there is a selection effect favoring the detection of such low-eccentricity systems: high eccentricities greatly increase the emission of gravitational radiation, and those systems quickly coalesce after their orbits have decayed (Chaurasia \& Bailes 2005). However, for the detection of young systems such as J1906+0746 that selection effect has not yet developed. Only 
binaries with eccentricities $e>0.94$ can expect to merge within J1906+0746's age of $\tau_{c}=112.6 \mathrm{kyr}$ (Peters 1964).

The low eccentricity and system velocity, combined with the relatively low mass of $\mathrm{J} 1906+0746$, suggest it was formed in an electron-capture, O-Ne-Mg supernova (van den Heuvel 2007). In such a case, the spin axis of the recycled pulsar is more likely to still be aligned with the orbital angular momentum, in which case it will show little geodetic precession (Section 8). This formation scenario can thus be falsified by a future detection of the recycled, companion pulsar if it does precess into view.

In conclusion, we currently cannot confirm with certainty or rule out that the companion of $\mathrm{J} 1906+0746$ is a NS; and given the fast decline in pulse flux due to geodetic precession, we will likely not improve on our timing solution until the pulsar precesses back into view. Pulsar J1906+0746 is likely in a binary containing a DNS; or it is orbited by a WD, in a system formed through an exotic binary interaction involving two stages of mass transfer.

We thank Jeroen Stil for advice on interpreting the VGPS data, Bryan Gaensler and Avinash Deshpande for discussions on the kinematic absorption method, and Lindley Lentati for comparing timing algorithms. The Arecibo Observatory is operated by SRI International under a cooperative agreement with the National Science Foundation (AST-1100968), and in alliance with Ana G. Méndez-Universidad Metropolitana, and the Universities Space Research Association. The National Radio Astronomy Observatory is a facility of the National Science Foundation operated under cooperative agreement by Associated Universities, Inc. The Westerbork Synthesis Radio Telescope is operated by ASTRON with support from the Netherlands Foundation for Scientific Research NWO. The Nançay radio Observatory is operated by the Paris Observatory, associated to the French Centre National de la Recherche Scientifique (CNRS). This work was supported by European Commission Grant FP7-PEOPLE-2007-4-3-IRG-224838 (J.v.L.) and by U.S. National Science Foundation Grants AST-0647820 (D.J.N.) and AST-0807556/AST-1312843 (J.M.W.). L.K. acknowledges the support of a doctoral Canada Graduate Scholarship. Pulsar research at UBC is supported by an NSERC Discovery Grant and by the Canada Foundation for Innovation.

\section{REFERENCES}

Alpar, M. A., Cheng, A. F., Ruderman, M. A., \& Shaham, J. 1982, Natur, 300,728

Amôres, E. B., \& Lépine, J. R. D. 2005, AJ, 130, 659

Antoniadis, J., Bassa, C. G., Wex, N., Kramer, M., \& Napiwotzki, R. 2011, MNRAS, 412, 580

Antoniadis, J., van Kerkwijk, M. H., Koester, D., et al. 2012, MNRAS, 423, 3316

Barker, B. M., \& O’Connell, R. F. 1975, ApJL, 199, L25

Bhat, N. D. R., Bailes, M., \& Verbiest, J. P. W. 2008, PhRvD, 77, 124017

Breton, R. P., Kaspi, V. M., Kramer, M., et al. 2008, Sci, 321, 104

Burgay, M., Joshi, B. C., D'Amico, N., et al. 2006, MNRAS, 368, 283

Champion, D. J., Lorimer, D. R., McLaughlin, M. A., et al. 2005, MNRAS, 363,929

Chatterjee, S., Brisken, W. F., Vlemmings, W. H. T., et al. 2009, ApJ, 698,250

Chaurasia, H. K., \& Bailes, M. 2005, ApJ, 632, 1054

Clifton, T. R., Frail, D. A., Kulkarni, S. R., \& Weisberg, J. M. 1988, ApJ, 333,332

Cordes, J. M., Freire, P. C. C., Lorimer, D. R., et al. 2006, ApJ, 637, 446

Cordes, J. M., \& Lazio, T. J. W. 2002, arXiv:astro-ph/0207156

Corongiu, A., Kramer, M., Stappers, B. W., et al. 2007, A\&A, 462, 703

Damour, T., \& Deruelle, N. 1986, AnIHP, 44, 263

Damour, T., \& Esposito-Farese, G. 1996, PhRvD, 54, 1474
Damour, T., \& Ruffini, R. 1974, CRASM, 279, 971

Damour, T., \& Taylor, J. H. 1991, ApJ, 366, 501

Damour, T., \& Taylor, J. H. 1992, PhRvD, 45, 1840

Deller, A. T., Bailes, M., \& Tingay, S. J. 2009a, Sci, 323, 1327

Deller, A. T., Brisken, W. F., Phillips, C. J., et al. 2011, PASP, 123, 275

Deller, A. T., Tingay, S. J., Bailes, M., \& Reynolds, J. E. 2009b, ApJ, 701,1243

Demorest, P. B. 2007, PhD thesis, Univ. California, Berkeley

Desvignes, G. 2009, PhD thesis, l'Université d'Orléans

Dewey, R. J., Taylor, J. H., Weisberg, J. M., \& Stokes, G. H. 1985, ApJL, 294, L25

Dicke, R. H. 1946, RScI, 17, 268

Dowd, A., Sisk, W., \& Hagen, J. 2000, in Pulsar Astronomy-2000 and Beyond, IAU Colloquium 177, ed. M. Kramer, N. Wex, \& R. Wielebinski (San Francisco, CA: ASP), 275

Ferdman, R. D. 2008, PhD thesis, Univ. British Columbia

Ferdman, R. D., Stairs, I. H., Kramer, M., et al. 2008, in AIP Conf. Proc. 983, 40 Years of Pulsars: Millisecond Pulsars, Magnetars and More, ed. C. Bassa, Z. Wang, A. Cumming, \& V. M. Kaspi (Melville, NY: AIP), 474

Ferdman, R. D., Stairs, I. H., Kramer, M., et al. 2014, MNRAS, 443, 2183

Fich, M., Blitz, L., \& Stark, A. A. 1989, ApJ, 342, 272

Fonseca, E., Stairs, I. H., \& Thorsett, S. E. 2014, ApJ, 787, 82

Frail, D. A., \& Weisberg, J. M. 1990, AJ, 100, 743

Freire, P. C. C., Wex, N., Esposito-Farèse, G., et al. 2012, MNRAS, 423, 3328

Fruchter, A. S., Bookbinder, J., \& Bailyn, C. 1995, ApJL, 443, L21

Haslam, C. G. T., Stoffel, H., Salter, C. J., \& Wilson, W. E. 1982, A\&AS, 47, 1

Hobbs, G., Lorimer, D. R., Lyne, A. G., \& Kramer, M. 2005, MNRAS, 360, 974

Hobbs, G., Lyne, A. G., Kramer, M., Martin, C. E., \& Jordan, C. 2004, MNRAS, 353,1311

Hobbs, G. B., Edwards, R. T., \& Manchester, R. N. 2006, MNRAS, 369, 655

Hotan, A. W., Bailes, M., \& Ord, S. M. 2005, ApJ, 624, 906

Hotan, A. W., van Straten, W., \& Manchester, R. N. 2004, PASA, 21, 302

Jacoby, B. A., Cameron, P. B., Jenet, F. A., et al. 2006, ApJL, 644, L113

Janssen, G. H., Stappers, B. W., Kramer, M., et al. 2008, A\&A, 490, 753

Johnston, S., Koribalski, B., Weisberg, J. M., \& Wilson, W. 2001, MNRAS, 322,715

Kaplan, D. L., Escoffier, R. P., Lacasse, R. J., et al. 2005, PASP, 117, 643

Karuppusamy, R., Stappers, B., \& van Straten, W. 2008, PASP, 120, 191

Kasian, L. 2008, in AIP Conf. Proc. 983, 40 Years of Pulsars: Millisecond Pulsars, Magnetars and More, ed. C. Bassa, Z. Wang, A. Cumming, \& V. M. Kaspi (Melville, NY: AIP), 485

Kasian, L. 2012, PhD thesis, Univ. British Columbia, https://circle.ubc.ca/handle/2429/41515

Kaspi, V. M., Lackey, J. R., Mattox, J., et al. 2000, ApJ, 528, 445

Keith, M. J., Kramer, M., Lyne, A. G., et al. 2009, MNRAS, 393, 623

Kramer, M. 1998, ApJ, 509, 856

Kramer, M., Stairs, I. H., Manchester, R. N., et al. 2006, Sci, 314, 97

Kramer, M., Wielebinski, R., Jessner, A., Gil, J. A., \& Seiradakis, J. H. 1994, A\&AS, 107, 515

Kramer, M., Xilouris, K. M., Lorimer, D. R., et al. 1998, ApJ, 501, 270

Kulkarni, S. R., \& van Kerkwijk, M. H. 1998, ApJL, 507, L49

Lorimer, D. R. 2008, LRR, 11, 8, http://relativity.livingreviews.org/Articles/ 1rr-2008-8/

Lorimer, D. R., \& Kramer, M. 2005, Handbook of Pulsar Astronomy (Cambridge: Cambridge Univ. Press)

Lorimer, D. R., Stairs, I. H., Freire, P. C., et al. 2006, ApJ, 640, 428

Lyne, A., Hobbs, G., Kramer, M., Stairs, I., \& Stappers, B. 2010, Sci, 329,408

Lyne, A. G., Burgay, M., Kramer, M., et al. 2004, Sci, 303, 1153

Manchester, R. N., Hobbs, G. B., Teoh, A., \& Hobbs, M. 2005, AJ, 129, 1993

Nice, D. J., \& Taylor, J. H. 1995, ApJ, 441, 429

Peters, P. C. 1964, PhRv, 136, 1224

Rafikov, R. R., \& Lai, D. 2006, PhRvD, 73, 063003

Reid, M. J., Menten, K. M., Brunthaler, A., et al. 2014, ApJ, 783, 130

Shklovskii, I. S. 1970, SvA, 13, 562

Stairs, I. H., Thorsett, S. E., \& Arzoumanian, Z. 2004, PhRvL, 93, 141101

Stappers, B. W., van Kerkwijk, M. H., Bell, J. F., \& Kulkarni, S. R. 2001, ApJL, 548, L183

Stil, J. M., Taylor, A. R., Dickey, J. M., et al. 2006, AJ, 132, 1158

Tauris, T. M., \& Sennels, T. 2000, A\&A, 355, 236

Taylor, J. H. 1987, in IAU Symp. 125, The Origin and Evolution of Neutron Stars, ed. D. J. Helfand \& J.-H. Huang (Dordrecht: Reidel), 383

Taylor, J. H., \& Cordes, J. M. 1993, ApJ, 411, 674

Taylor, J. H., \& Weisberg, J. M. 1989, ApJ, 345, 434

Thorsett, S. E., Arzoumanian, Z., McKinnon, M. M., \& Taylor, J. H. 1993, ApJL, 405, L29 
Tutukov, A., \& Yungelson, L. 1993, MNRAS, 260, 675

van den Heuvel, E. P. J. 2007, in AIP Conf. Proc. 924, The Multicolored Landscape of Compact Objects and Their Explosive Origins, ed. T. di Salvo,

G. L. Israel, L. Piersant, L. Burderi, G. Matt, A. Tornambe, \& M. T. Menna (Melville, NY: AIP), 598

van Kerkwijk, M., \& Kulkarni, S. R. 1999, ApJL, 516, L25

van Leeuwen, J., Cordes, J. M., Lorimer, D. R., et al. 2006, CJAAS, 6, 020

van Straten, W., \& Bailes, M. 2011, PASA, 28, 1

van Straten, W., Demorest, P., \& Oslowski, S. 2012, AR\&T, 9, 237
Verbiest, J. P. W., Weisberg, J. M., Chael, A. A., Lee, K. J., \& Lorimer, D. R. 2012, ApJ, 755, 39

Voûte, J. L. L., Kouwenhoven, M. L. A., van Haren, P. C., et al. 2002, A\&A, 385,733

Weisberg, J. M., Boriakoff, V., \& Rankin, J. 1979, A\&A, 77, 204

Weisberg, J. M., Nice, D. J., \& Taylor, J. H. 2010, ApJ, 722, 1030

Weisberg, J. M., Romani, R. W., \& Taylor, J. H. 1989, ApJ, 347, 1030

Weisberg, J. M., Stanimirović, S., Xilouris, K., et al. 2008, ApJ, 674, 286

Will, C. M. 1993, Theory and Experiment in Gravitational Physics (Cambridge: Cambridge Univ. Press) 